June 26, 2015

\title{
The current impact flux on Mars and its seasonal variation
}

\author{
Youngmin JeongAhn and Renu Malhotra \\ Lunar and Planetary Laboratory, The University of Arizona, Tucson, AZ 85721, USA. \\ jeongahn@lpl .arizona.edu, renu@lpl .arizona.edu
}

\begin{abstract}
We calculate the present-day impact flux on Mars and its variation over the Martian year, using the current data on the orbital distribution of known Marscrossing minor planets. We adapt the Öpik-Wetherill formulation for calculating collision probabilities, paying careful attention to the non-uniform distribution of the perihelion longitude and the argument of perihelion owed to secular planetary perturbations. We find that, at the current epoch, the Mars crossers have an axial distribution of the argument of perihelion, and the mean direction of their eccentricity vectors is nearly aligned with Mars' eccentricity vector. These previously neglected angular non-uniformities have the effect of depressing the mean annual impact flux by a factor of about 2 compared to the estimate based on a uniform random distribution of the angular elements of Mars-crossers; the amplitude of the seasonal variation of the impact flux is likewise depressed by a factor of about 4-5. We estimate that the flux of large impactors (of absolute magnitude $H<16$ ) within $\pm 30^{\circ}$ of Mars' aphelion is about three times larger than when the planet is near perihelion. Extrapolation of our results to a model population of meter-size Mars-crossers shows that if these small impactors have a uniform distribution of their angular elements, then their aphelion-to-perihelion impact flux ratio would be 11-15, but if they track the orbital distribution of the large impactors, including their non-uniform angular elements, then this ratio would be about 3. Comparison of our results with the current dataset of fresh impact craters on Mars (detected with Mars-orbiting spacecraft) appears to rule out the uniform distribution of angular elements.
\end{abstract}

\section{Introduction}

The impact crater record on the surfaces of the terrestrial planets over geologically long timescales provides a window on the dynamical history of the solar system, including 
a chronology of major geological and dynamical events. This crater-based chronology is calibrated primarily on estimates of the cratering rate on the Moon over geological timescales. The presence of space observatories in orbit about Mars now offers a new opportunity to measure the present-day impact flux on Mars in statistically significant numbers, at least for small impactors, and thereby improve the calibration of crater-based chronology for geologically recent times. Within the last $\sim$ decade, almost 500 dated impact sites have been identified, by comparison of before- and after- images of Mars' surface (Malin et al. 2006; Daubar et al. 2015), including some that can be narrowed down to within a half Martian year (Daubar et al. 2012). Also over the past $\sim$ decade, large scale and deep surveys for near-Earth objects have dramatically increased our knowledge of the population of minor planets in the inner solar system, hence the size and orbital distribution of the current Martian impactor population can be identified. Thus, it is valuable to investigate the theoretically expected Martian impact rate based on the known impactor population, and to compare it with observations of contemporary Martian impacts.

The impact rate on Mars has been the subject of several previous studies. Ivanov (2001) adapted the lunar impact crater production function to calculate the long-term impact crater production rate on Mars. A series of studies (Hartmann \& Neukum 2001; Hartmann 2005; Chapman 2004; Hartmann et al. 2010, and references therein) have calculated increasingly refined estimates of Martian cratering, taking account of complexities associated with secondary cratering and other effects. Le Feuvre \& Wieczorek (2008) and Le Feuvre \& Wieczorek (2011) used a model of the orbital distribution of planet-crossing objects to calculate the Martian cratering rate. Williams et al. (2014) used the observed annual flux of terrestrial fireballs to calculate the present-day production rate of small Martian craters. In many of these studies, the Mars/Moon ratio of impact cratering is a fundamental parameter, and the studies attempt to obtain the impact crater production rate averaged over geological timescales for use in crater-based chronologies.

In the present paper, we make an independent and improved estimate of the current impact flux on Mars, based on the known observational data set of the present-day population of Mars crossing objects (MCOs). We demonstrate that the MCOs have an intrinsically nonrandom distribution of the longitude of perihelion $\varpi$ and argument of perihelion $\omega$, owing to secular planetary perturbations. Such non-uniformity has been noted previously: the non-uniform $\omega$ circulation cycle of MCOs was briefly discussed as a protection mechanism against collisions on Mars (Wetherill 1974). The concentration of $\varpi$ in the asteroid belt was investigated for its role in the impact flux amongst main belt asteroids and Hildas (Dell'Oro \& Paolicchi 1998; Dell'Oro et al. 2001). However, detailed study of the non-uniform distribution of the angular elements of MCOs has not been carried out, nor its effects on the impact rate on Mars. Therefore, we first identify the non-uniformities in the distribution of 
$\varpi$ and $\omega$ in the unbiased population of bright MCOs, then we develop a numerical method for computing the impact probabilities that takes account of these non-uniformities. We demonstrate that these non-uniformities have a significant effect on both the mean impact flux as well as its variation over Mars' seasons. Our calculations are based on a modification of the method of Wetherill (1967) and Öpik (1951) which computes the probability of collision of two objects that move on fixed, independent Keplerian orbits. The modification that we introduce accounts directly for the non-randomness of the angular elements of MCOs. We also account in detail for gravitational focusing effects; these are also significantly affected by the non-random distribution of the angular elements of MCOs because the encounter velocities of MCOs with Mars are correlated with their angular elements.

The rest of this paper is organized as follows. In Section 2, we describe the observational data set of MCOs, derive an observationally complete subset, and describe the dynamically significant aspects of the distribution of its orbital elements. In Section 3, we describe the details of our impact flux calculations and present our results. In Section 4, we provide a comparison of our results with the results of previous studies, and in Section 5, we summarize our results.

\section{Mars Impactor Population}

\subsection{Definitions and Dynamical Considerations}

To estimate the current impact flux on Mars, we need to know the intrinsic orbital distribution of current Mars Crossing objects (MCOs), free from observational selection effects. The orbital distribution is defined on the parameter space of the five orbital elements, semimajor axis, eccentricity, inclination, argument of perihelion and longitude of perihelion, $(a, e, i, \omega, \varpi)$. We obtain this by first looking to the current data on MCOs, and using a brightness cutoff to define a subset that is less affected by observational bias; we also estimate a correction factor for the observational completeness of this subset. We adopt the orbital distribution of the bright MCOs as a proxy for the orbital distribution of the entire population of Mars impactors. It is conceivable that the orbital distribution of the population of very small MCOs differs from that of the bright (large) MCOs; we discuss this point in Section 4.5.

The current population of MCOs can be defined with cutoffs in perihelion and aphelion distances, analogous to the common definition of Near Earth Objects (NEOs). Thus, the aphelion of MCOs should be greater than the current perihelion distance of Mars, 1.38 AU, and their perihelion should be smaller than the current aphelion of Mars, $1.67 \mathrm{AU}$. We 
obtained the current data on all minor planets from the Minor Planet Center (MPC), which maintains up-to-date lists of the minor planets of the solar system. The dataset "MPCORB" on 15 June 2014 contains the osculating orbital elements as well as the absolute brightness magnitude of more than $6 \times 10^{5}$ minor planets. (We do not include objects that have poorly determined orbit solutions from only a single opposition of observation.) Of these, only 16702 objects meet the definition of MCOs.

It is of some importance to note that the orbital parameters of Mars (and of the planet-crossing population of minor planets) vary significantly over geological timescales. On megayear timescales, Mars' orbital eccentricity varies between near-zero and $\sim 0.12$, and its orbital inclination also varies from near zero to $\sim 8$ degrees relative to the invariable plane of the Solar system (Ito \& Tanikawa 2002; Laskar et al. 2004). The current eccentricity, 0.093, is closer to its long term maximum value, whereas the current inclination, 1.85 degrees to the ecliptic (1.67 degrees to the invariable plane), is closer to its minimum value. The current semimajor axis of Mars is $1.524 \mathrm{AU}$; over megayear timescales, the semimajor axis variation is only $0.03 \%$ from maximum to minimum (determined from our own numerical integration for a timespan of $100 \mathrm{myr}$ ). Mars' perihelion longitude, $\varpi$, is currently at $336^{\circ}$ and its longitude of ascending node, $\Omega$, is at $50^{\circ}$, with respect to the ecliptic and equinox of J2000, and their current rates of change are 0.43 and -0.28 degrees per century, respectively (Murray \& Dermott 1999).

Most previous studies have used estimates of the long-term average value of the impact rate to draw isochrones (e.g., Williams et al. 2014). This rate is time-variable as Mars' eccentricity varies on secular timescales, therefore, the current value of Mars' eccentricity, 0.093, should be used when the current impact rate is of interest. We are focusing on the current impact rate on Mars in this paper but the sensitive nature of the impact rate with respect to time-varying Martian eccentricity will be briefly discussed later, in Section 5.2.

Martian climate seasons are conventionally defined by its vernal and autumnal equinoxes and according to the solar longitude, $L_{S}$, in reference to its obliquity. However, as we will see below, Mars' variable heliocentric distance is a major factor that determines the annual variation of the impact flux. Therefore, the "seasonal variation" of the impact flux on Mars is logically defined with respect to Mars' perihelion longitude, which corresponds to $L_{S}=251^{\circ}$. At the current era, the northern hemisphere Winter roughly corresponds to the time of perihelion of Mars. 

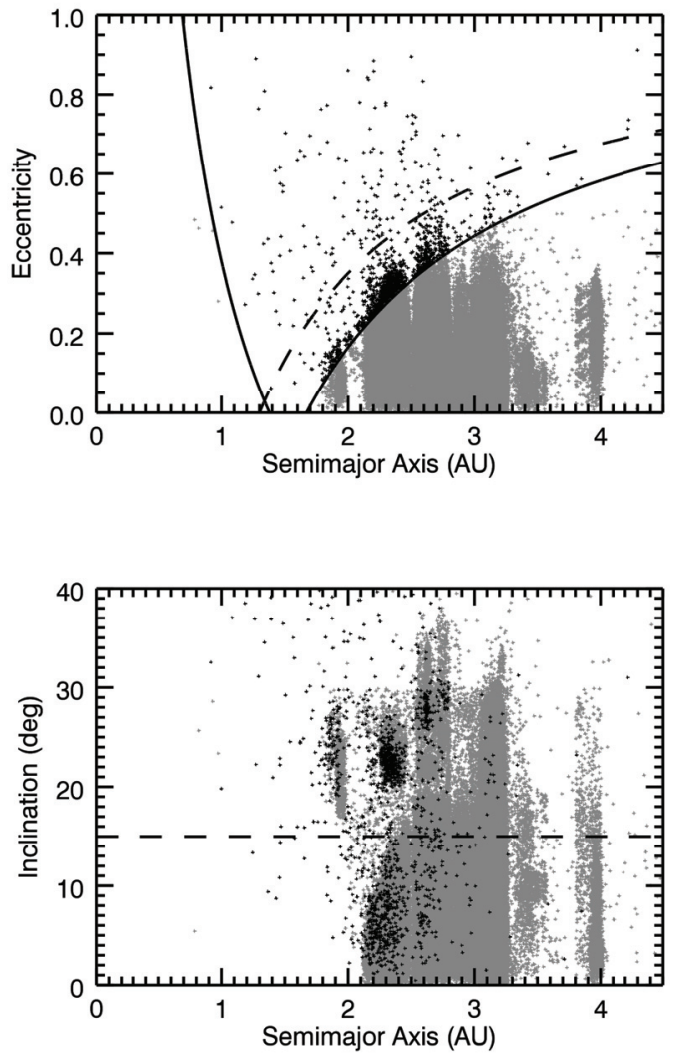

Fig. 1.- Orbital distribution of bright $(H<16)$ minor planets in the inner solar system. The horizontal axes denote semimajor axes and the vertical axes are eccentricity and inclination in the upper and lower panel, respectively. Mars crossers are marked as black points, all others are in gray. The continuous line curves in the upper panel define the inner and outer limits of Mars crossers; the dashed curve indicates the outer boundary of Amors at perihelion distance $q=1.3 \mathrm{AU}$. In the lower panel, the boundary of the "high inclination" and "low inclination" subgroups is indicated with the horizontal dashed line at $i=15^{\circ}$. 


\subsection{Orbital distribution of MCOs}

A scatter plot of the semimajor axes and eccentricities of bright MCOs, of absolute magnitude $H<16$, is shown in the upper panel of Fig. 1. Most of this sample (about $85 \%$ of 1322 objects) has semimajor axes in the range between $2.1 \mathrm{AU}$ and $3.3 \mathrm{AU}$; note that this range overlaps with the main asteroid belt. In this sample, 167 (13\%) objects have perihelion distance smaller than 1.3 AU and therefore also belong to the population of minor planets defined as near-Earth objects. The number of bright MCOs, $H<16$, that actually cross Earth's orbit (perihelion distance $q<1 \mathrm{AU}$ ) is $84(6 \%)$. The small body database of Jet Propulsion Laboratory (JPL) excludes NEOs from MCOs, for classification purposes; this was also done in a classification study of MCOs, Michel et al. (2000). However, in the work we carry out here, we do not discard the current Earth-crossing population from MCOs because this population also contributes to the current Martian impact statistics.

A scatter plot of the semimajor axes, $a$, and inclinations, $i$, is shown in the lower panel of Fig. 1. In this plot, a clear division between "low inclination" and "high inclination" subsets of MCOs is evident, with a boundary at $i=15^{\circ}$. A finer set of divisions was defined by Michel et al. (2000) who classified the MCOs into five different categories based on the three orbital parameters $(a, e, i)$. According to the definitions of Michel et al. (2000), the dividing line of

$i=15^{\circ}$ corresponds to the lower inclination boundary of the Hungaria group of asteroids and it roughly matches with the location of the $\nu_{6}$ secular resonance which separates the Phocaea asteroid group and the highly inclined outer main belt asteroids (MB2, in their notation) from the main belt asteroids of low inclination ( $\mathrm{MB}$, in their notation). Here we distinguish only the two broader groups, "low inclination" and "high inclination" MCOs, with the boundary at $i=15^{\circ}$. As we will show, these two groups differ systematically in the distributions of their angular elements as well as in their impact velocities and their probabilities of collision with Mars.

\subsection{Absolute magnitude distribution and completeness of MCOs}

The absolute brightness magnitude of MCOs ranges from $H=9.4$ (132 Aethra) to $H=33.2$ (2008 TS26), however the population of fainter objects is highly incomplete due to many observational selection effects. Figure 2 plots the cumulative $H$ magnitude distribution of MCOs up to $H=18$. The MPC database lists $H$ magnitudes with only one decimal place accuracy in most cases; for the binned distribution, the MCOs corresponding to boundary values were allotted equally to adjacent bins. We observe that the slope of the $H$ distribution decreases noticeably for $H>15$, indicating increasing observational incompleteness; therefore, we assume that MCOs are observationally complete for $H<15$. 


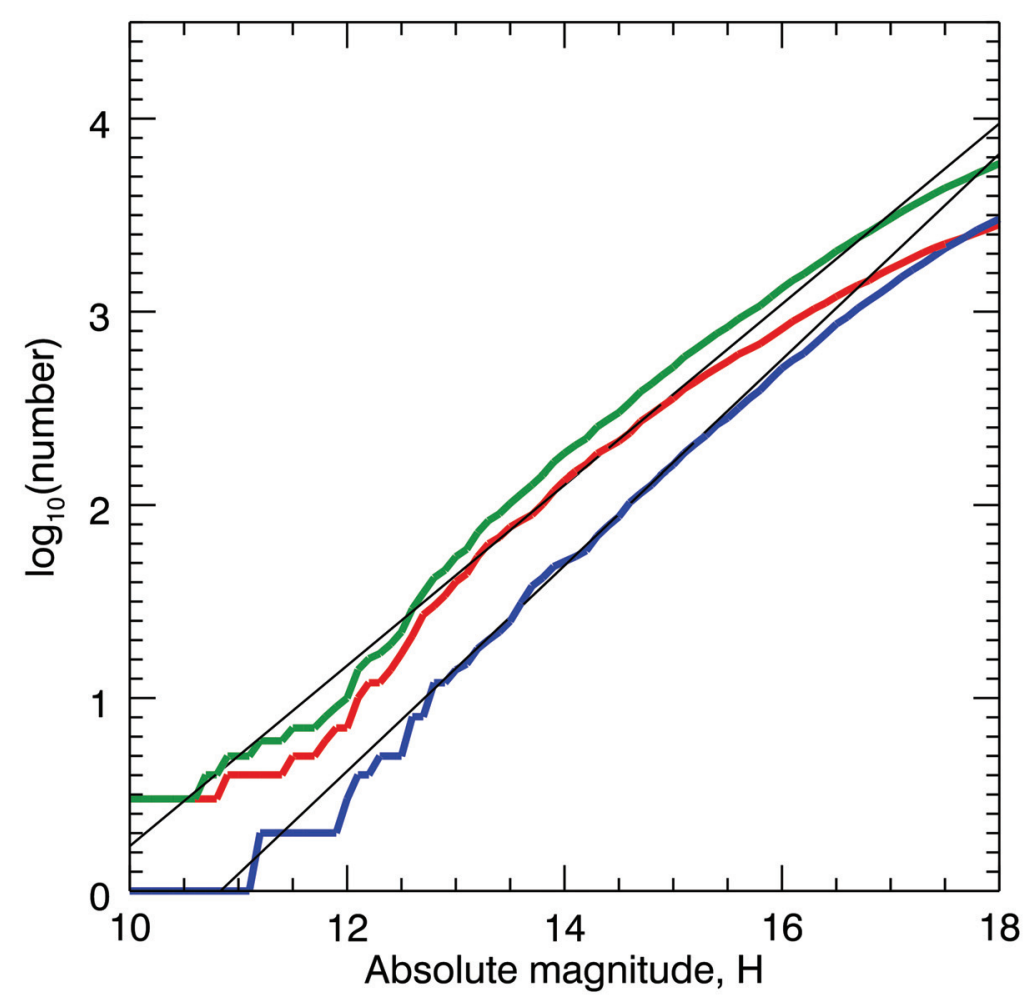

Fig. 2.- Cumulative distribution of the absolute magnitude, $H$, of known Mars crossing objects. Note that the ordinate is on a logarithmic scale. The green curve indicates all MCOs, the red and blue curves indicate the high inclination $\left(i>15^{\circ}\right)$ and the low inclination $\left(i<15^{\circ}\right)$ subsets. The two black diagonal lines indicate the best-fit linear functions for high and low inclination MCOs with $13<H<15$. 
However, there are only 516 known MCOs with $H<15$. To obtain better statistics for their orbital distribution, we adopted the sample of MCOs having $H<16$; the uncertainty caused by this choice is discussed in Section 4.3. This sample consists of 1322 objects ( $8 \%$ of the 16702 known MCOs with well-determined orbits).

To calculate the incompleteness level of our sample of bright MCOs, we estimated their intrinsic number as follows. We observe in Fig. 2, that both the low (blue curve) and high (red curve) inclination MCOs follow an almost straight line, hence a single power law distribution, in the range $13<H<15$. We calculated the least-squares best-fit linear function for the cumulative distribution of MCOs in this brightness range. The best-fit slope of the low inclination population, $0.533 \pm 0.009$, is found to be slightly higher than that of the high inclination population, $0.468 \pm 0.007$. (We quote $1-\sigma$ uncertainties of the best-fit slopes.) Because the difference in slopes of the low and high inclination groups is more than $7-\sigma$, we assume that it is real.

(We also calculated the best-fit linear function for the smaller range, $13<H<14$. The slopes of both populations are steeper in this range compared to the slopes over the 13-15 $H$-magnitude range, but again the low inclination MCOs have a steeper slope, $0.603 \pm 0.020$, than the high inclination ones, $0.504 \pm 0.018$. The cause of the steeper slope within $H$ of 13-14 compared to $H$ within 13-15 could be the wavy character of the size and brightness distribution, but undetected objects near $H$ of 15 can also contribute to this trend.) A steeper slope at low inclinations was also found for main belt asteroids (Terai \& Itoh 2011).

With the above best-fits, we can describe the cumulative number distribution of MCOs of brightness $H \gtrsim 13$ as

$$
N(<H)=A_{L} \times 10^{\alpha_{L}(H-15)}+A_{H} \times 10^{\alpha_{H}(H-15)},
$$

where the first term, with $A_{L}=166 \pm 4$ and $\alpha_{L}=0.533 \pm 0.009$, describes the low inclination MCOs, and the second term, with $A_{H}=373 \pm 7$ and $\alpha_{H}=0.468 \pm 0.007$, describes the high inclination MCOs.

With this approximation, we estimate that the total number of bright MCOs $(H<16)$ is 1662, and the observed sample of 1322 represents $80 \%$ of this intrinsic population. Therefore, the undetected MCOs $(H<16)$ are about $11 \%$ and $35 \%$ of the observed MCOs, in the low inclination and the high inclination subgroups, respectively.

We note that we have implicitly assumed that the $H$ distribution of bright MCOs is largely independent of their orbital distribution (save for the inclinations, as discussed). However, the $H$ magnitude distribution may vary in orbital parameter space due to sizedependent local collisional history or non-gravitational effects such as the thermal Yarkovsky drift. The different power law distributions of the high and low inclination subgroups of 
MCOs we calculated above reflect part of this complex reality. Another example of this complexity is the $\mathrm{V}$-shaped dispersion of the Hungaria family in the $a-H$ diagram due to Yarkovsky drift (Warner et al. 2009).

We understand also that the Hungarias have higher average albedos than other MCOs due to a high fraction of E-type asteroids (Cañada-Assandri et al. 2015). Therefore, by choosing a single $H$ magnitude cutoff, our sample includes smaller size Hungarias than in the other populations. However, the number of Hungarias in our sample is 113, accounting for only $8.5 \%$ of the bright MCOs; therefore we keep using the single magnitude cutoff, $H<16$.

Later in this paper (sections 4.4, 4.5 and 5.1) we will also have need of an estimate of the population of smaller/fainter MCOs. It is difficult to assess how well Eq. 1 represents the population of fainter MCOs, $H \gtrsim 16$. It is well known that the size distribution of NEOs and of main belt asteroids both have a wavy shape (O'Brien \& Greenberg 2005). Thus, using a single slope approximation, as Eq. 1, can lead to an erroneous estimate for fainter MCOs $(H>16)$. Considering that current knowledge of the $H$ distribution of MCOs is limited, an equally good approximation for the fainter MCOs is to assume that the shape of their $H$ magnitude distribution follows that of the better-known NEOs, with a simple scale factor,

$$
N(<H)=N(<16) \times \frac{N_{\text {neo }}(<H)}{N_{\text {neo }}(<16)} .
$$

We obtained the brightness distribution function of NEOs, $N_{n e o}(<H)$, as follows. Le Feuvre \& Wieczorek (2011) provide a 10th-order polynomial fit to a normalized size distribution of NEOs down to sub-meter sizes, based on various telescopic observational data of NEOs and atmospheric bolides data (Halliday et al. 1996; Rabinowitz et al. 2000; Revelle 2001; Brown et al. 2002; Harris 2002; Morbidelli et al. 2002; Stuart \& Binzel 2004). We adopt this polynomial function, together with a mean albedo of 0.13 (Stuart \& Binzel 2004) to convert NEO diameter to $H$ magnitude, to calculate the ratio $N_{\text {neo }}(<H) / N_{\text {neo }}(<16)$. Finally, by adopting our estimate of the number of MCOs of $H<16, N(<16)=1096$ and $N(<16)=$ 566 for the high inclination and low inclination subgroups, respectively, Eq. 2 provides an alternative to Eq. 1 to estimate the population of smaller MCOs of these two inclination subgroups.

\subsection{Non-uniform Apsidal Distribution}

The distribution of the argument of perihelion, $\omega$, of bright MCOs is shown in Fig. 3. The MCOs' $\omega$ distribution is not independent of their inclinations. For the high inclination 
MCOs $\left(i>15^{\circ}\right.$, sample size $N=813$ objects) the concentrations around 90 and 270 degrees are evident. One possible reason for this concentration is the $i-e-\omega$ coupling caused by Jupiter's perturbation (Kozai 1962). This coupling, which occurs preferentially for high inclination orbits, causes the eccentricity to reach a maximum when $\omega$ is near $90^{\circ}$ or $270^{\circ}$. The maxima in eccentricity enable many of the high inclination objects to just barely be classified as MCOs. Thus, the boundary of MCOs in parameter space selectively contributes to the $\omega$ concentration. Secondly, long residence times around $\omega \simeq 90^{\circ}$ and $270^{\circ}$ (due to the slow rate of change of $\omega$ near these values) may also contribute to the concentrations.

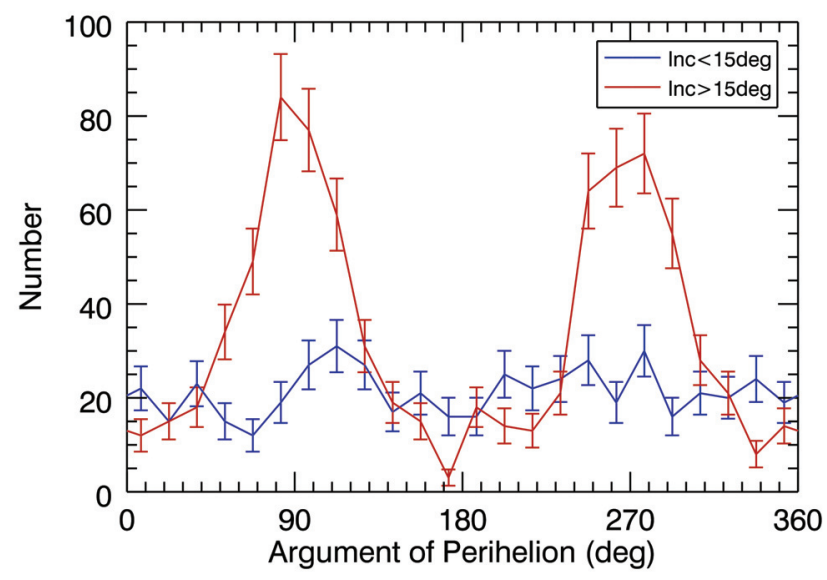

Fig. 3.- The distribution of the argument of perihelion, $\omega$, of bright MCOs $(H<16)$, in two different inclination regimes.

The coupled oscillations of $i-e-\omega$ are illustrated in the orbital evolution of 132 Aethra, the brightest and possibly the first discovered Mars' crossing asteroid. Its current orbital elements, $(a, e, i)=\left(2.608,0.389,25^{\circ}\right)$, are rather typical of the high inclination bright MCOs. We integrated 132 Aethra's orbit over a period of $10^{5}$ years, including the gravitational perturbations of all eight planets. We found that its semimajor axis variation is about $0.3 \%$, but its eccentricity and inclination exhibit large amplitude variations of opposite phase, with a period of about $10^{4}$ years, as shown in Fig. 4a. The large eccentricity of Aethra at the present epoch makes it a Mars-crosser, but lower eccentricity over time changes this circumstance. The maxima in the eccentricity occur at $\omega=90^{\circ}$ and $\omega=270^{\circ}$, as shown in Fig. 4b. In this figure, we also highlight the range when Aethra's perihelion distance is less than Mars' aphelion distance; we observe that this occurs when its argument of perihelion is near $\omega=90^{\circ}$ and $\omega=270^{\circ}$. Further study is needed to confirm that this is the dominant 
behavior amongst high inclination MCOs.
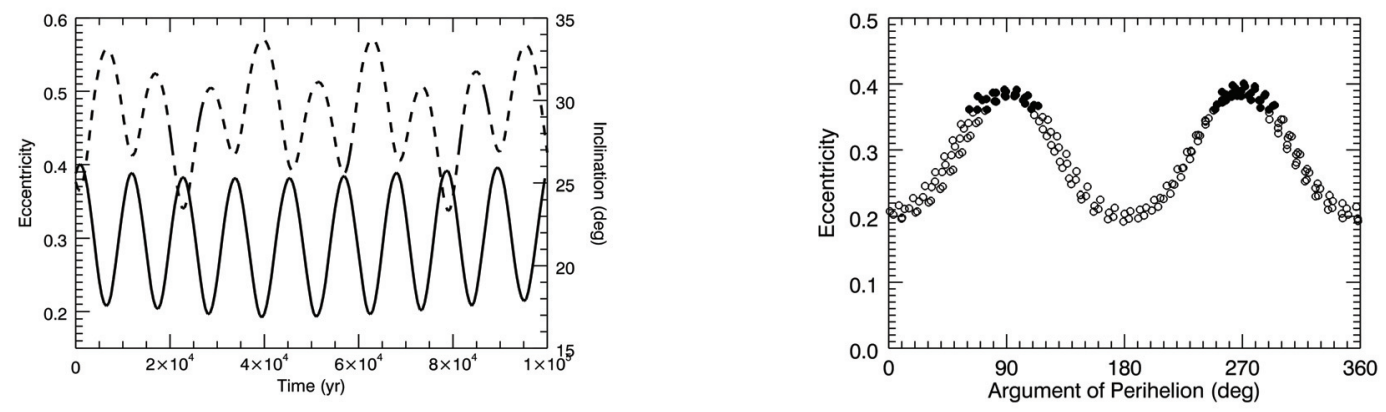

Fig. 4. - The orbital evolution of 132 Aethra over $10^{5}$ years, including the gravitational perturbations from the planets. (a) The evolution of eccentricity (solid line) and inclination (dashed line). (b) The coupling between eccentricity and argument of perihelion; the filled circles indicate the times when Aethra's perihelion distance is less than Mars' aphelion distance.

In some contrast with the high inclination subset of MCOs, the low inclination subset $\left(i<15^{\circ}\right.$, sample size $N=509$ ) does not show as clear an axial distribution of $\omega$. We calculate the mean direction of $\omega$ and its significance level based on the statistical methods for axial distributions (Fisher 1993). We find that the mean direction is along $\bar{\omega}=101^{\circ}$ and $281^{\circ}$; however, the Rayleigh test shows that the non-uniformity is not statistically significant; the probability $p$ that the distribution is uniform on the circle is greater than $5 \%$. Therefore, for the currently available observational data set, we conclude that the low inclination MCOs are uniformly distributed in argument of perihelion.

The longitude of perihelion, $\varpi$, of bright MCOs has a strong unidirectional concentration, as seen in Fig. 5. This intrinsic concentration is also caused by secular effects due to planetary perturbations. For main belt asteroids as well as most MCOs, the secularly forced eccentricity vector is directed approximately along the perihelion direction of Jupiter, $\varpi_{4}=15^{\circ}$. Using Laplace-Lagrange secular theory (Murray \& Dermott 1999), we calculate that the forced eccentricity vector is directed towards $6^{\circ}$ ecliptic longitude for semimajor axis $a=3 \mathrm{AU}$; it gradually decreases to $344^{\circ}$ for $a=2.3 \mathrm{AU}$ and to $305^{\circ}$ for $a=2 \mathrm{AU}$ where the forced eccentricity vector becomes discontinuous (in the vicinity of the $\nu_{6}$ secular resonance). Because the apsidal precession rate is slowest when an asteroid's free eccentricity vector is aligned with the forced eccentricity vector, the non-uniform precession rate over secular timescales leads to the non-uniform distribution of perihelion longitude. Similar non-uniformities are found for the Amors (JeongAhn \& Malhotra 2014, see their Figures $14-16)$. 


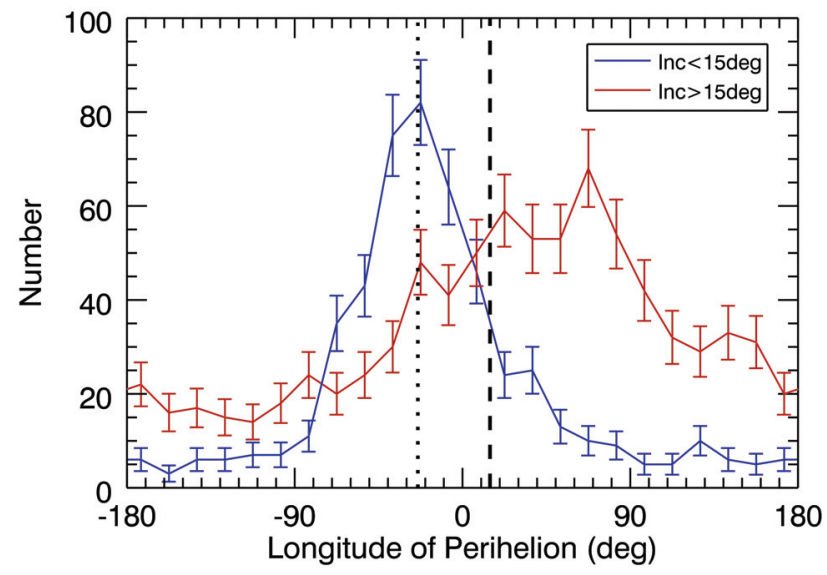

Fig. 5. - The distribution of the longitude of perihelion, $\varpi$, of bright MCOs $(H<16)$, in two different inclination regimes. The current value of the longitude of perihelion of Mars, $\varpi_{\sigma}$, and of Jupiter, $\varpi_{4}$, are indicated by the vertical dotted and dashed lines, respectively.

The peak direction and the degree of concentration of the $\varpi$ distribution are both different for the high and low inclination MCOs (Fig. 5). The low inclination MCOs $\left(i<15^{\circ}\right)$ have a narrow peak centered at $\bar{\varpi}=341^{\circ}$ while the high inclination MCOs $\left(i>15^{\circ}\right)$ show a broader peak centered at $\bar{\varpi}=48^{\circ}$. The non-uniformity of $\varpi$ distribution of the MCOs plays an important role in the seasonal variation of the impact frequency on Mars.

Mars' longitude of perihelion also generally shares the secular behavior of the low inclination MCOs; its apsidal precession rate is slowest when $\varpi_{\sigma^{\pi}} \approx \varpi_{J}$ (where it has correspondingly longer residence time). As noted above in Section 2.1, at the present time, Mars' eccentricity is close to its long term maximum; its apsidal precession rate is close to its long term minimum, and its current longitude of perihelion, $\varpi_{\sigma^{7}} \simeq 336^{\circ}$, is also close to the peak values of both MCO populations. This near-coincidence has significant implications for the current impact rate on Mars because some orbital geometries of MCOs can prohibit impact on Mars. We illustrate this in Fig. 6, in which we trace the elliptical orbit of Mars and the orbits of two representative $\mathrm{MCO}$ orbits. The two MCO orbits have the same semimajor axis and eccentricity, which we chose to be median values of the bright MCO $(H<15)$ sample, $a=2.39 \mathrm{AU}, e=0.345$, but one has its perihelion aligned with Mars' $\left(\varpi \approx \varpi_{\sigma^{\pi}}\right)$ while the other has perihelion anti-aligned with Mars' $\left(\varpi \approx \varpi_{0^{\pi}}+180^{\circ}\right)$. It is obvious that the former prohibits impact on Mars. Because Mars' current perihelion direction coincides with the direction of high concentration of the MCOs' perihelia, the intrinsic impact rate 


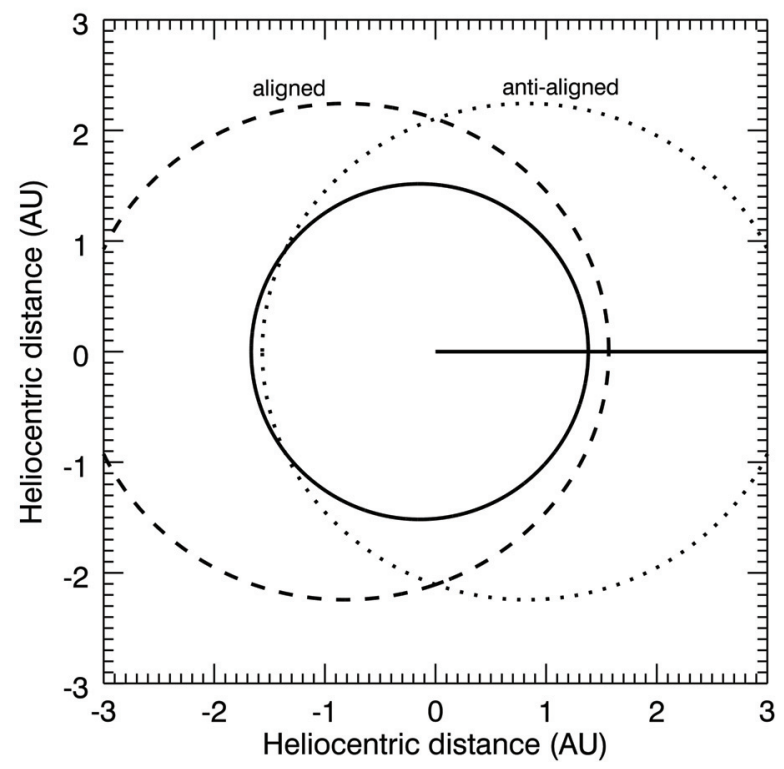

Fig. 6. - Schematic diagram of Mars' orbit (black solid ellipse) and two possible asteroid orbits having the same semimajor axis and eccentricity but having different perihelion longitudes, one aligned with Mars' perihelion (dashed curve) and the other aligned with Mars' aphelion (dotted curve). The direction of Mars' perihelion is indicated by the solid horizontal line starting from the location of the Sun at the origin. The semimajor axis and eccentricity of the asteroids were chosen as the median values of the bright MCOs ( $H<15), a=2.39 \mathrm{AU}$ and $e=0.345$. For simplicity, all the orbits are assumed to have zero inclination from the projected plane.

can be significantly lower compared to the rate calculated from an assumed uniform random distribution of $\varpi$. Previous studies have usually adopted a uniform random distribution of $\varpi$.

The longitude of ascending node, $\Omega$, of MCOs is uniformly distributed, as shown in Figure 7 for both the low-inclination and the high-inclination populations. The Rayleigh test for uni-directionality for the bright MCOs indicates that their $\Omega$ distribution is indistinguishable from a uniform distribution $(p>0.05)$. Even though minor intrinsic non-uniformities might be present due to secular dynamics (because the normal to Mars' orbital plane deviates from that of the invariable plane as well as from the local secularly forced inclination vector of MCOs), a minor non-uniformity would not have a significant effect on the impact rate. 


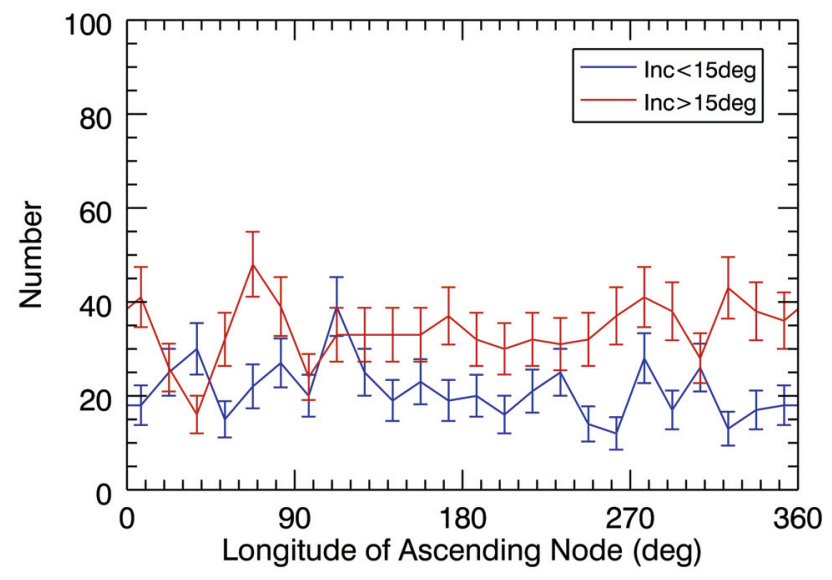

Fig. 7. - The distribution of the longitude of ascending node, $\Omega$, of bright MCOs $(H<16)$, in two different inclination regimes. Both groups are statistically indistinguishable from a uniform distribution.

\section{Modified Öpik-Wetherill Method}

We first briefly describe the calculation of the probability per unit time that an MCO in a given orbit approaches Mars within a prescribed encounter distance, then we describe our method for the generation of "clones" of MCOs, and finally our method for calculating the impact flux on Mars.

We adopt the method pioneered by Öpik (1951) and Wetherill (1967) for the calculation of collision probability, $P$, between two objects on fixed Keplerian orbits. While Öpik (1951)'s method restricted one of the colliding pair to a circular orbit, Wetherill (1967) generalized this method to two eccentric orbits having arbitrary mutual inclination and apsidal orientations. A number of subsequent works have adopted Wetherill's equation for $P$ and provided improved methods for computing its mean value over secular precession timescales (Greenberg 1982; Bottke \& Greenberg 1993; Pokorný \& Vokrouhlický 2013).

Consider a particular location of Mars in its orbit. Following Wetherill (1967), the probability per unit time for an MCO to approach Mars within a prescribed distance $\tau$ can be calculated in terms of the relative velocity vector and the orbital periods of the two orbits. We express Wetherill's formula in simpler notation as

$$
P(\tau)= \begin{cases}\pi \tau\left(2 U_{0} T T_{0} \sqrt{1-\left(\mathbf{U} \cdot \mathbf{U}_{\mathbf{0}}\right)^{2} U^{-2} U_{0}^{-2}}\right)^{-1} & \text { if } \tau>D_{\min }, \\ 0 & \text { if } \tau<D_{\min }\end{cases}
$$


where $D_{\min }$ is the minimum distance between the orbit of the MCO and the orbit of Mars, $\mathbf{U}_{0}$ is the heliocentric velocity of Mars at the encounter, $\mathbf{U}$ is the encounter velocity of the MCO relative to Mars, $T$ is the orbital period of the MCO, and $T_{0}$ is the orbital period of Mars. This expression for $P(\tau)$ forms the backbone for our impact flux calculations. The dependence of $P(\tau)$ on the orbital elements of Mars and of the MCO is implicit in the velocities, $\mathbf{U}, \mathbf{U}_{0}$, in the right hand side of Eq. 3. Note that this probability does not account for any gravitational focusing effect of Mars, which we will address below.

Previous calculations (Öpik 1951; Wetherill 1967; Greenberg 1982; Bottke \& Greenberg 1993) commonly adopted uniform distributions of the angular orbital elements by assuming uniform angular precession rates to calculate impact probabilities. However, this is not justified for MCOs, as shown in the previous section. Furthermore, these distributions differ for the high and low inclination subgroups of MCOs. Therefore, we carry out impact flux calculations separately for each subgroup.

In order to avoid the small number statistics problem, we generate clones having the current orbital distribution of MCOs, as follows. For each MCO within each inclination subgroup, we generate ten thousand clones by taking each triad, $(a, e, i)$ and assigning to it a value of $\omega$ and of $\varpi$ chosen randomly from the $\omega$ 's and $\varpi$ 's of the respective inclination subgroup of MCOs. In this way, we preserve the correlations amongst $(a, e, i)$ as well as the respective non-random distributions of $\omega$ and of $\varpi$ of the high and low inclination subgroups, while generating a sufficient number of clones to obtain good statistics for the impact flux calculations. We understand that, even though we subdivided the MCOs into two distinct inclination groups, there could be residual correlations between $\omega, \varpi$ and the other orbital elements; we neglect this complication here.

For each MCO clone, we calculate the Minimum Orbit Intersection Distances (MOIDs) between Mars and the clone, with the code developed by Gronchi (2005). This distance is defined as the closest possible distance between any random locations on each orbit. The MOID is a widely used measure for assessing the impact hazard of near-Earth objects on Earth but can be used with any two osculating orbits. We identify a possible collision between two orbits if their MOID is less than a prescribed small distance, $D$. We use $D=0.001 \mathrm{AU}$, which corresponds to 44 times the physical radius of Mars, $R_{\mathrm{O}^{7}}$. (A correction factor, $\left(R_{O^{x}} / D\right)^{2}$, is applied later (see below) to convert to actual collisional statistics.) We record the velocity vectors of the $\mathrm{MCO}$ and of Mars at the MOID location, to enable the calculation of collision probability, $P(D)$, with Eq. 3. We also record the longitude where MOID occurs, to track the seasonal variation of the impact rate. It is theoretically possible that two orbits have two "crossings" at two nodes. However, such cases are very rare, and we neglect these rare possibilities, recording only a single position where MOID occurs. 
Now, among all the MCO clones, only a small fraction meets the criterion of MOID $<D$. These MCOs would potentially approach Mars closer than the small distance $D$ in the near future (absent any orbit changes, e.g. owing to planetary perturbations). For each of these potential Mars colliders, we calculate the probability $P(D)$ with the use of Eq. 3 . Then, the impact rate of the bright MCOs of absolute magnitude $H<H_{l i m}$ is computed as follows:

$$
I\left(H_{\text {lim }}\right)=\frac{(1+b) R_{\sigma^{\Upsilon}}^{2}}{D^{2} N_{c}} \sum_{i}\left(1+\frac{2 G M_{\sigma^{\Upsilon}}}{R_{\sigma^{\top}} U_{i}^{2}}\right) P_{i}(D),
$$

where the sum is over all the cloned population of MCOs and $N_{c}=10^{4}$ is the clone multiplicity. There are three correction factors included above: $(1+b)$ accounts for the observational incompleteness of our sample of bright MCOs (in Section 2.3, we estimated $b=0.35$ for high inclination MCOs and $b=0.11$ for the low inclination ones, respectively, for $H_{\text {lim }}=16$ ); the factor $\left(R_{\mathrm{O}^{\top}} / D\right)^{2}$ accounts for the inflated collision cross section in our calculation of $P_{i}(D)$; the factor $\left(1+2 G M_{\sigma^{7}}\left(R_{\sigma^{7}}^{-1} U_{i}^{-2}\right)\right.$ accounts for the gravitational enhancement of the collision cross section of Mars for an MCO having encounter velocity $U_{i}$.

The impact flux on Mars is defined as the number of impacts per unit time per unit surface area, i.e. $F=I /\left(4 \pi R_{\sigma^{\top}}^{2}\right)$, therefore,

$$
F\left(H_{\text {lim }}\right)=\frac{(1+b)}{4 \pi D^{2} N_{c}} \sum_{i}\left(1+\frac{2 G M_{\sigma^{\top}}}{R_{\bigcirc^{\top}} U_{i}^{2}}\right) P_{i}(D) .
$$

Because we also recorded the longitude where the MOID occurs for each cloned MCO, we can calculate the impact flux on Mars as a function of its orbital longitude (measured from Mars' perihelion direction), as follows. Consider the longitude bin $\left\{\lambda_{j}-\frac{1}{2} \Delta \lambda, \lambda_{j}+\frac{1}{2} \Delta \lambda\right\}$. The impact rate in this bin is given by

$$
F_{j}\left(H_{\text {lim }}\right)=\frac{(1+b) T_{0}}{4 \pi D^{2} N_{c} \Delta T_{j}} \sum_{i}\left(1+\frac{2 G M_{\sigma^{\nearrow}}}{R_{\sigma^{\nearrow}} U_{i}^{2}}\right) P_{i}(D) \delta_{i j},
$$

where $\delta_{i j}$ is unity when the impact site of the $i$-th clone is located within the $j$-th longitude bin, and zero otherwise, and $\Delta T_{j} / T_{0}$ is the fraction of its orbital period that Mars spends in the $j$-th longitude bin. The following relation holds by definition,

$$
F\left(H_{\text {lim }}\right)=\sum_{j} F_{j}\left(H_{\text {lim }}\right) \frac{\Delta T_{j}}{T_{0}} .
$$

In order to assess the statistical uncertainty in our impact flux estimates, we carry out the impact flux calculations with three independent realizations of the randomly generated 
clone sets. In the results reported below, we quote the average over these three sets. The standard deviation of these three realizations is our estimate of the statistical uncertainty due to the random generation of clones; we find this to be on the order of a few percent of the average. Additionally, in order to illustrate the role of the non-uniformity of angular distributions, we also generate a "control set" of clones by assigning uniform random values to $\omega$ and $\varpi$, and we carry out the impact flux calculation separately with this set.

\section{Results}

\subsection{Impact flux on Mars}

The impact flux and its seasonal variation are calculated following the steps explained in the previous section. As noted, we carried out calculations for the high inclination and the low inclination groups of MCOs separately, and then combined the two sets. The detailed results are listed in Table 1. For comparison, the results from the case of uniform angular distribution are also listed in Table 1 . In addition, we intentionally neglected the gravitational focusing in order to illustrate the role of gravitational focusing in Martian impact statistics; we list the results so obtained in the last two rows in the Table. For all of these listed results, we report the average of three simulations; the standard deviations of the fluxes obtained in these simulations are all below $3.5 \%$ of the reported flux. We describe first our results for the mean impact flux (F, Eq. 5) and then our results for the seasonal variation of the impact flux $\left(F_{j}\right.$, Eq. 6). The distribution of impact velocity and the effect of gravitational focusing will be described afterwards.

We find that the overall mean impact flux, $F(16)$ (Eq. 5), is $2.9 \times 10^{-23} \mathrm{~km}^{-2} \mathrm{~s}^{-1}$. The contributions to this mean flux by the two inclination subgroups are similar: $1.4 \times$ $10^{-23} \mathrm{~km}^{-2} \mathrm{~s}^{-1}$ and $1.5 \times 10^{-23} \mathrm{~km}^{-2} \mathrm{~s}^{-1}$ for the high inclination MCOs and the low in-

Table 1: The impact flux of bright MCOs $(H<16)$ on Mars and its seasonal variation.

\begin{tabular}{|c|c|c|c|c|c|}
\hline \multirow[t]{2}{*}{$\omega, \varpi$ distribution } & \multicolumn{3}{|c|}{$\begin{array}{l}\text { mean impact flux, } F(16) \\
\qquad\left(10^{-23} \mathrm{~km}^{-2} \mathrm{~s}^{-1}\right)\end{array}$} & \multicolumn{2}{|c|}{ aphelion-to-perihelion flux ratio } \\
\hline & all & $i>15^{\circ}$ & $i<15^{\circ}$ & $\pm 30^{\circ}$ & half Martian year \\
\hline Non-uniform & 2.9 & 1.4 & 1.5 & 3.0 & 2.1 \\
\hline \multirow[t]{2}{*}{ Uniform } & 6.1 & 2.4 & 3.8 & 11.0 & 5.4 \\
\hline & \multicolumn{5}{|c|}{ Gravitational focusing neglected } \\
\hline Non-uniform & 2.1 & 1.2 & 0.9 & 2.6 & 1.9 \\
\hline Uniform & 4.1 & 2.0 & 2.1 & 8.1 & 4.4 \\
\hline
\end{tabular}


clination groups, respectively. Although the population of the high inclination subgroup is twice as large as the low inclination subgroup, the low intrinsic impact probabilities of higher inclination MCOs reduces their contribution to the impact statistics. It is noteworthy that the impact rates are significantly higher when we adopt a uniform random distribution of the angular elements $(\omega, \varpi)$ : the impact flux for the high inclination group is larger by a factor 1.7 and the flux for the low inclination group is larger by a factor 2.4; the overall rate is 2.1 times higher than for the non-uniform case. The difference is larger for the low inclination group. We can understand these trends with the help of the schematic diagram in Figure 6 which illustrates that MCOs whose eccentricity vectors are aligned with Mars' eccentricity vector are less likely to impact Mars. For the high inclination group, the axial distribution of $\omega$ also plays an important role in reducing the impact flux because these MCOs tend to reach perihelion when they are located off the orbital plane of Mars.

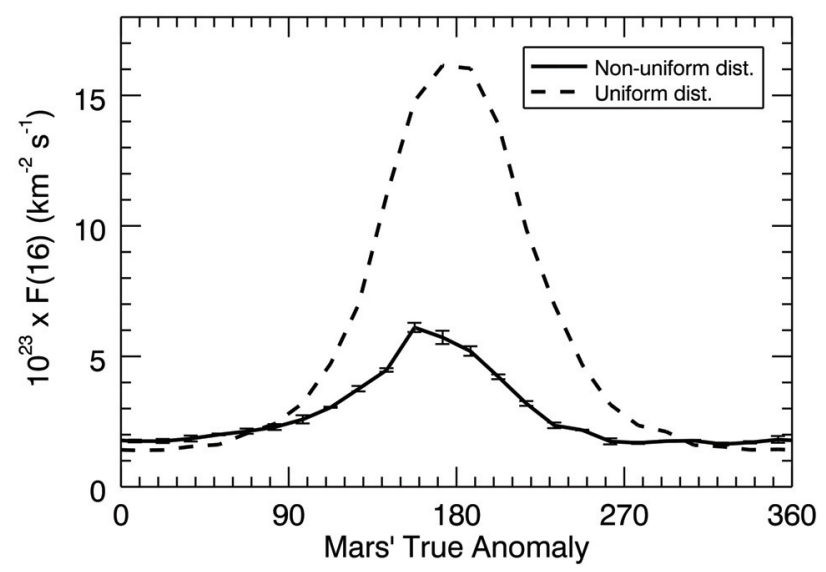

Fig. 8. - (Solid line) The seasonal variation of the impact flux, $F_{j}(16)$ (Eq. 6), of the bright MCOs $(H<16)$; for each longitude bin, we calculated the mean from three independent realizations of the randomly generated clones of MCOs; the error bars indicate the standard deviation of the three simulations. (Dashed line) For comparison, we also computed the impact flux with an assumed uniform distribution of the angular elements $(\omega, \varpi)$ of MCOs. The longitude bin size is 15 degrees.

The results for the seasonal variation in the impact flux are shown in Figures 8 and 9, in which we plot the total impact flux, and the impact flux contributed by the low inclination MCOs and the high inclination MCOs, respectively, as a function of Mars' true anomaly, i.e., $F_{j}$ (Eq. 6), with 15-degree longitude bins. The continuous line curves plot the results for the case of the non-uniform distribution of the angular elements $(\omega, \varpi)$ of the bright MCOs. 


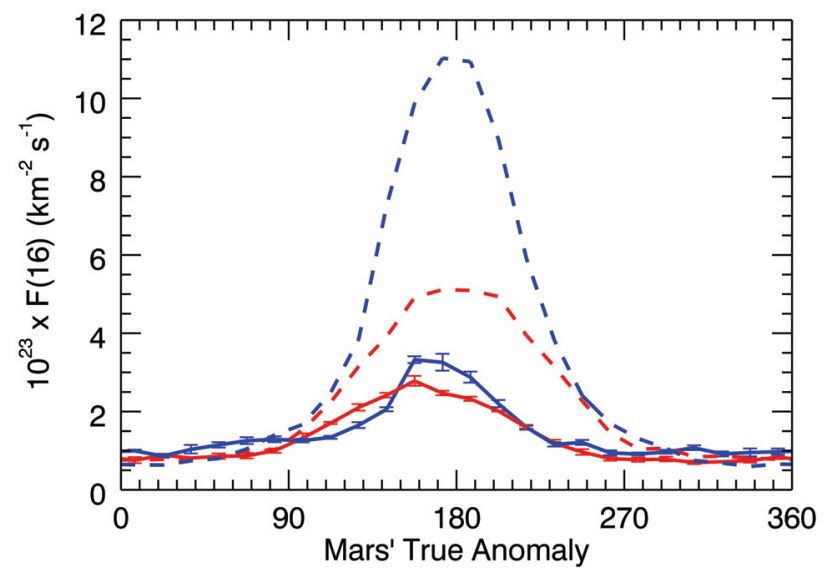

Fig. 9.- Same as Figure 8, but limited to the high inclination subgroup, $i>15^{\circ}$ (red), and the low inclination subgroup, $i<15^{\circ}$ (blue).

For comparison, we also plot the result for an assumed uniform random distribution of these angles (dashed line). We observe that the impact flux on Mars peaks when the planet is near aphelion and MCOs are at their perihelion. There are at least two reasons for this: the orbit-crossing geometry (see Figure 6) is more favorable, and the spatial density of MCOs increases with heliocentric distance. A third reason is due to the lower encounter velocity (hence larger gravitational cross section) of MCOs; this is described below in Section 4.2.

It is also evident that the non-uniform distribution of the angular elements significantly affects the amplitude of the seasonal variability of the impact flux. To express this quantitatively, we calculated the impact flux over the true anomaly range of $\pm 30^{\circ}$ centered at Mars' aphelion and its perihelion. Then we find that the aphelion-to-perihelion ratio of the impact flux is 11.0 for the case of the uniform distribution but it is only 3.0 for the actual non-uniform distribution. Another way to illustrate this is with the half-year ratio: integrating over half a Martian year centered at the planet's aphelion, we find that impacts are 2.1 times more frequent than during the half Martian year centered at perihelion. In contrast, this ratio is 5.4 if we assume uniform random distribution of the angular elements. The non-uniform distribution of the MCOs' longitude of perihelion, with a concentration near Mars perihelion, means that the orbital geometry that favors impact conditions (Figure 6) occurs less frequently (compared to the case of a uniform random distribution of $\varpi$ ), thereby diminishing the seasonal concentration of the impacts near Mars' aphelion and also diminishing the overall impact flux. 
We remark that the high and low inclination subgroups have a similar seasonal variation of the impact flux when we take account of the non-uniform angular distribution. However, we find a significant difference in their seasonal variation in the simulations with uniform angular distributions: the $\left( \pm 30^{\circ}\right)$ aphelion-to-perihelion ratio is about 6.4 for the high inclination subgroup and about 16 for the low inclination subgroup. This may be of importance for the impact flux of small MCOs which may be dominated by low inclinations and possibly more uniform angular distribution due to non-gravitational effects. We discuss this further in Section 4.5.

\subsection{Impact velocities}

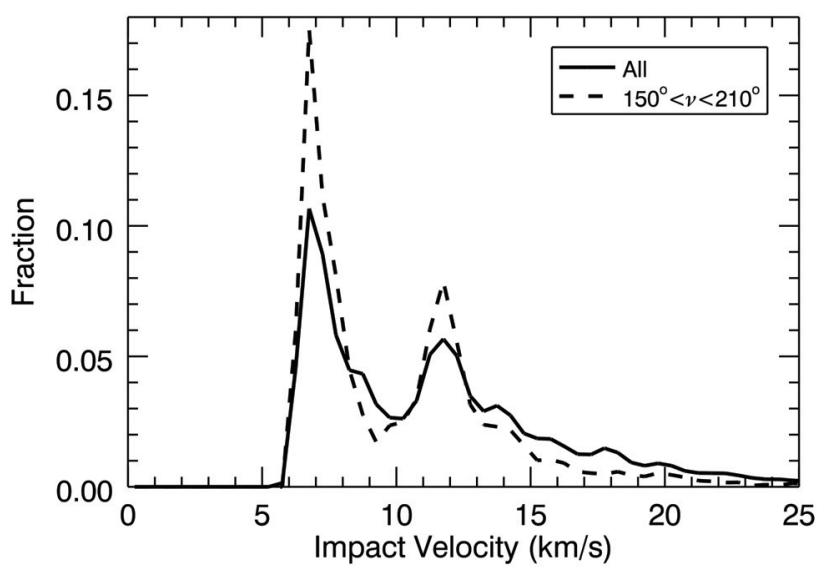

Fig. 10. - The solid line plots the distribution of impact velocity of bright MCOs with Mars, the dashed line plots the impact velocity distribution near Mars' aphelion (true anomaly in the range $150^{\circ}$ to $210^{\circ}$ ). We accounted for the effects of the intrinsic collision probability of individual MCOs as well as the different observational completeness levels between the high inclination population and low inclination population.

As mentioned previously, in our simulations we tracked the encounter velocities, $U$, of MCOs with Mars. We computed the impact velocities as $v_{i m p}^{2}=U^{2}+2 G M_{\sigma^{7}} R_{\sigma^{7}}^{-1}$. In Figure 10, we plot the distribution of impact velocity of MCOs with Mars integrated over the Martian year (solid line curve), and the impact velocity distribution when the planet is near aphelion (dashed curve). For the latter, we integrated over the true anomaly range of $\pm 30^{\circ}$ around the aphelion of Mars. 
The mean encounter velocity is $10.1 \mathrm{~km} / \mathrm{s}$, equivalent to a mean impact velocity of $11.5 \mathrm{~km} / \mathrm{s}$. (The simulations with uniform random distribution of the angular elements give slightly lower values, $8.5 \mathrm{~km} / \mathrm{s}$ and $10.1 \mathrm{~km} / \mathrm{s}$, respectively.) The impact velocity distribution has two distinct peaks, at $\sim 6.5 \mathrm{~km} / \mathrm{s}$ and $\sim 11.5 \mathrm{~km} / \mathrm{s}$. The lower velocity peak is contributed by low inclination MCOs, and the higher velocity peak is by high inclination ones, because of their larger vertical component of the relative velocity. This justifies our choice in separating the two groups with the inclination boundary at $i=15^{\circ}$. (These two peaks can be also noticed in the results of Le Feuvre \& Wieczorek (2011), in their Fig. 4.) The overall encounter velocity distribution is similar to the distribution from aphelion colliders (shown as the dashed curve in Figure 10) because the aphelion collisions dominate the impact flux.

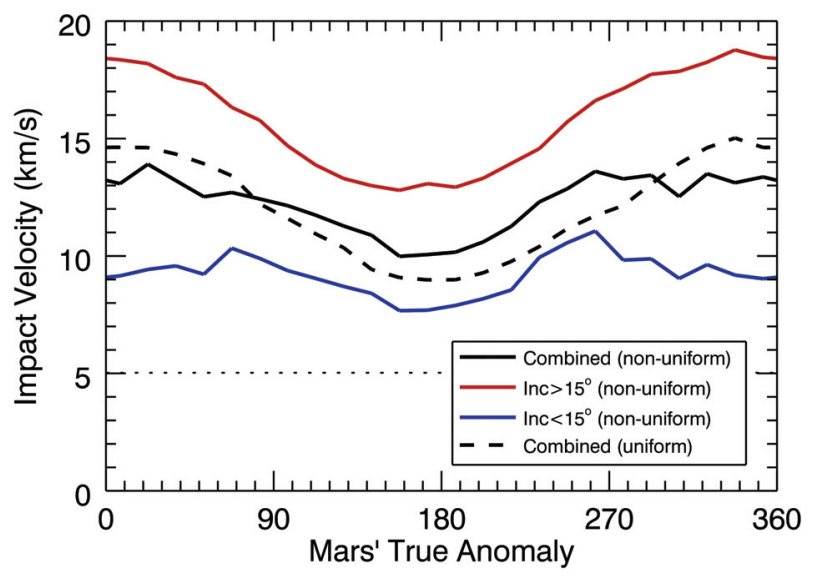

Fig. 11. - Mean impact velocity of bright MCOs $(H<16)$ and its seasonal variation. The red and blue curves plot the mean impact velocity of the high inclination subgroup $\left(i>15^{\circ}\right)$ and the low inclination subgroup $\left(i<15^{\circ}\right)$, respectively, and the continuous black curve plots the combined mean encounter velocity of all bright MCOs. For comparison, the dashed black curve shows the mean impact velocity resulting from the uniform distribution of angular elements. The dotted horizontal line indicates the escape velocity of Mars, $v_{e s c}=5 \mathrm{~km} / \mathrm{s}$.

The impact velocities are seasonally variable. In Figure 11, we plot the mean impact velocity of MCOs with Mars, as a function of Mars' true anomaly (with 15-degree longitude bins). The mean impact velocity of the high and low inclination subgroups of MCOs as well as that of the combined population are plotted. The mean impact velocity is lowest near Mars' aphelion and highest when the planet is near perihelion. The seasonal variation is larger for the high inclination group. Our calculations also show that the seasonal variation for the case of non-uniform angular distribution (black solid line) is subdued compared to 
the case of uniform random angular distribution (black dashed line).

The encounter velocity affects the gravitational focusing factor in the impact flux calculation. Table 1 shows that gravitational focusing not only increases the mean impact flux by $40 \%$ but also its seasonal variability. The low mean encounter velocity near Mars' aphelion enhances the collision cross section more than near perihelion, and this is an additional factor that enhances the impact flux near Mars' aphelion. Also it is evident that the effect of gravitational focusing is much more significant for low inclination MCOs due to their lower encounter velocities, accounting for about $70 \%$ increased impact flux.

\subsection{Observational sample bias}

The results described above are based on the orbital distribution of the bright $(H<16)$ MCOs, together with an estimate of their completeness. We repeated the calculations, for the MCOs with a brighter magnitude cut-off, $H<15$, for which the known population is more complete. This allows us to examine whether the observational bias in the orbital distribution of fainter objects has an effect on our results. There are only 516 known MCOs having $H<15$. With this smaller set, the detailed results varied from the results from $H<16$ objects, but we still find the same ratio, 2.1, of the impact flux in the half-Martian year centered at the planet's aphelion versus perihelion. The mean impact velocity, 11.6 $\mathrm{km} / \mathrm{s}$, also matches the result from $H<16$ objects. We find their mean impact flux is $F(15)=7.6 \times 10^{-24} \mathrm{~km}^{-2} \mathrm{~s}^{-1}$. Extrapolating to the fainter population of $H<16$ MCOs by use of Eq. 1, we then estimate $F(16)=2.5 \pm 0.4 \times 10^{-23} \mathrm{~km}^{-2} \mathrm{~s}^{-1}$. This is within about $15 \%$ of our simulation result (see Table 1), and it indicates the uncertainty owed to the observational sample bias.

\subsection{Mars/Moon and Mars/Earth impact flux ratios}

Studies of crater chronology based on impact crater densities often use estimates of the impact flux ratio of Mars and the Moon. Additionally, in the next section we will make use of the impact flux ratio of Mars and the Earth. We therefore applied the same method as above to calculate the impact flux of bright Earth-crossing objects (ECOs) on the Moon and Earth.

Making use of the same source of minor planet data (the MPCORB dataset), we identified the 516 bright ECOs having $H<18$; these are assumed to be observationally complete (Mainzer et al. 2011; JeongAhn \& Malhotra 2014). Most of this population of ECOs is the 
Apollo subgroup of NEOs. The Apollos have a strongly axial distribution of the argument of perihelion, concentrated near 0 and 180 degrees, but their longitude of ascending node is statistically indistinguishable from a uniform random distribution (JeongAhn \& Malhotra 2014). Therefore, we took account of the non-uniformity in $\omega$ and carried out the calculation of the impact flux for the Earth and the Moon in the same way as we did for the Martian impact flux. We included gravitational focusing for individual encounters, but for the lunar impact flux we neglected the orbital velocity of the Moon about the Earth. Our result for the mean impact flux on the Moon is $F_{D}(18)=6.06 \times 10^{-23} \mathrm{~km}^{-2} \mathrm{~s}^{-1}$ and for the Earth it is $F_{\oplus}(18)=1.06 \times 10^{-22} \mathrm{~km}^{-2} \mathrm{~s}^{-1}$; the Earth/Moon impact flux ratio is 1.7 .

To obtain the Mars/Moon and Mars/Earth impact flux ratios, we extrapolate our Martian impact flux estimate, $F(16)$, to the fainter magnitude, $H<18$, by using a multiplication factor, $N(<18) / N(<16)$. We calculate $N(<18)$ and $N(<16)$ from Eq. 1 , and obtain $F(18)=3.0 \times 10^{-22} \mathrm{~km}^{-2} \mathrm{~s}^{-1}$ for the Martian impact flux. From this, we find that the current Mars/Moon impact flux ratio is 5.0, and the Mars/Earth impact flux ratio is 2.8. However, as noted in section 2.3, Eq. 1 may overestimate the number of fainter MCOs. If instead we adopt Eq. 2 for the $H$ distribution of fainter MCOs, we obtain a somewhat smaller impact flux, $F(18)=1.8 \times 10^{-22} \mathrm{~km}^{-2} \mathrm{~s}^{-1}$. The impact flux ratios are also smaller: 2.9 for Mars/Moon and 1.7 for Mars/Earth. In Section 5.1, we compare these results with previous estimates in the literature.

\subsection{Flux of small impactors}

The flux of small impactors, in the size range 1-10 meters, is of interest because these are likely to be frequent enough that space telescopes currently orbiting Mars would be able to observe them as fresh craters on the surface of the planet. Impactors of this size range that enter Earth's atmosphere are sometimes referred to as "bolides" in the literature. The orbital distribution of such small MCOs is not certain, as they may or may not track the orbital distribution of the bright MCOs. (Their long term orbital evolution is likely to be affected by the Yarkovsky effect. However, the mean semimajor axis drift rate for small MCOs of diameter $D \lesssim 30 \mathrm{~m}$ is quite small, $|\dot{a}| \lesssim 0.01$ AU per million years (Farinella et al. 1998; O’Brien \& Greenberg 2005).) If the orbital distribution of small MCOs is the same as that of the bright MCOs, then we can straightforwardly predict that the seasonal variation of their current impact flux will be similar, i.e., a maximum impact flux at aphelion, and a ratio of about 3 for the flux at $\pm 30^{\circ}$ aphelion-to-perihelion. Should observations find a significantly different (larger) seasonal variation of fresh craters on Mars, it would point to differences in the orbital distribution of the small MCOs relative to the large MCOs. For 
example, a uniform distribution of the angular parameters of small impactors would produce a much larger aphelion-to-perihelion flux ratio, as Table 1 suggests.

Here we extend our impact flux calculation to meter-size MCOs, using three different estimates of the population of small MCOs. In the first, we extrapolate the $H$-magnitude distribution of the bright MCOs (Eq. 1) down to $H$ magnitude of 33 which corresponds to approximately meter-size objects. We note that our model of the cumulative $H$-magnitude distribution as a combination of two power law functions, with different slopes for the low and high inclination subgroups, suggests that, at the faint magnitude of $H \approx 33$, the low inclination objects are about 7 times more numerous than high inclination objects. Admittedly, this is a very large extrapolation; a small change in the power law slope, e.g., by $\sim 0.1$ in the range of $H=16-33$, can lead to a factor of $\sim 50$ over- or under-estimate of the cumulative population of $H<33$ MCOs. Nevertheless, we bravely proceed, and we find that the low and high inclination subgroups imply a flux of $\sim 1.8 \times 10^{-14} \mathrm{~km}^{-2} \mathrm{~s}^{-1}$ and $\sim 1.3 \times 10^{-15} \mathrm{~km}^{-2} \mathrm{~s}^{-1}$, respectively, for an estimated total bolide flux on Mars of $F(33) \approx 1.9 \times 10^{-14} \mathrm{~km}^{-2} \mathrm{~s}^{-1}$.

A second way to estimate the number of small MCOs is with the use of Eq. 2, which is scaled from the $H$-distribution of NEOs (and assumes that the orbital distribution of small MCOs is the same as that of the bright MCOs). We find the number ratio of $\left[N_{\text {neo }}(<\right.$ $\left.33) / N_{\text {neo }}(<16)\right] \approx 5.5 \times 10^{7}$. Then multiplying our computed value of $F(16)$ with this factor we find $F(33) \approx 1.6 \times 10^{-15} \mathrm{~km}^{-2} \mathrm{~s}^{-1}$.

For a third estimate, we look to the observational data for the bolide flux at Earth and use our estimate of the Mars/Earth impact flux ratio. Brown et al. (2002) have reported the cumulative size distribution of terrestrial bolides as

$$
\log _{10} N_{B}(>D)=1.568-2.70 \log _{10} D
$$

where $D$ is the bolide diameter in meters, and $N_{B}(>D)$ is the average number of bolides per year. This indicates about 37 impacts per year on Earth for $D>1 \mathrm{~m}$ (equivalently, $H \lesssim 33$ ) objects, or $F_{\oplus}(33) \approx 2.3 \times 10^{-15} \mathrm{~km}^{-2} \mathrm{~s}^{-1}$ in our notation. This estimate is consistent with more recent bolide data (Brown et al. 2013). Then, making use of the Mars/Earth impact flux ratio of 1.7-2.8 that we calculated in the previous section (for $H<18$ ), and assuming that this ratio is preserved down to meter-size objects, yields a Martian bolide flux of $F(33) \approx(3.7-6.4) \times 10^{-15} \mathrm{~km}^{-2} \mathrm{~s}^{-1}$. This estimate lies in-between the two estimates obtained above.

To summarize, the above three estimates find that the bolide flux on Mars is $F(33) \approx$ $(1.6-19) \times 10^{-15} \mathrm{~km}^{-2} \mathrm{~s}^{-1}$. The three estimates span a factor of up to 12 , which can be considered unsurprising and acceptable, given the large uncertainties in extrapolating the 
Table 2: The impact flux of meter-size MCOs $(H<33)$ on Mars and its seasonal variation.

\begin{tabular}{|c|c|c|c|c|c|}
\hline \multirow[t]{2}{*}{$\omega, \varpi$ distribution } & \multicolumn{3}{|c|}{$\begin{array}{l}\text { mean impact flux, } F(33) \\
\qquad\left(10^{-15} \mathrm{~km}^{-2} \mathrm{~s}^{-1}\right)\end{array}$} & \multicolumn{2}{|c|}{ aphelion-to-perihelion flux ratio } \\
\hline & all & $i>15^{\circ}$ & $i<15^{\circ}$ & $\pm 30^{\circ}$ & half Martian year \\
\hline & \multicolumn{5}{|c|}{ Population estimate from Eq. 1} \\
\hline Non-uniform & 19 & 1.3 & 18 & 3.1 & 2.0 \\
\hline \multirow[t]{2}{*}{ Uniform } & 45 & 2.2 & 43 & 15 & 6.6 \\
\hline & \multicolumn{5}{|c|}{ Population estimate from Eq. 2} \\
\hline Non-uniform & 1.6 & 0.8 & 0.8 & 3.0 & 2.1 \\
\hline Uniform & 3.4 & 1.3 & 2.1 & 11 & 5.4 \\
\hline
\end{tabular}

MCO population from $H \approx 16$ to $H \approx 33$. These mean impact flux estimates imply that Mars suffers $14-160$ impacts by $D>1$ m objects per Martian year. An impact rate of this magnitude may be detectable in the rate of fresh craters.

The first two estimates are tabulated in Table 2, including separate contributions from the two inclination subgroups. As before, for comparison, we also calculated the impact fluxes for the case of uniform distribution of angular elements. We see that neglecting nonuniformities of the angular elements increases the mean impact flux by a factor of 2, similar to the case of bright MCOs (Section 4.1, Table 1).

We also report in Table 2 the seasonal variations of the impact flux of the meter size objects, for both population models, Eq. 1 and Eq. 2. The population model of Eq. 2 predicts that the seasonal variation of faint objects $(H>16)$ does not depend on the $H$ magnitude (in this model, the number ratio between the two inclination subgroups is fixed). Therefore, the aphelion-to-perihelion impact flux ratios obtained with this model are identical to the results in Table 1 for the bright MCOs. For the population model of Eq. 1, we expect that the meter-size impactors are dominated by low inclination orbits, then the seasonal variation can be expected to be similar to that of the low inclination subgroup. As the high and low inclination subgroups have a similar seasonal variation for the non-uniform distribution of angular elements (Fig. 9), the combined seasonal variation is found to be almost the same as the case of bright $\operatorname{MCOs}(H<16)$, i.e., the $\left( \pm 30^{\circ}\right)$ aphelion-to-perihelion impact flux ratio is $\sim 3.1$ and the half Martian year impact flux ratio is $\sim 2.0$. Notably, however, the case of uniform distribution of angular elements leads to a larger seasonal variation: the $\left( \pm 30^{\circ}\right)$ aphelion-to-perihelion impact flux ratio is $\sim 15$ and the half Martian year impact flux ratio is $\sim 6.6$. 


\section{Discussion}

\subsection{Comparisons with previous studies}

For the large impactors $(D \gtrsim 1 \mathrm{~km}$ or $H \lesssim 18$ ), there are two important previous studies to which we can compare our results, Ivanov (2001) and Le Feuvre \& Wieczorek (2008). The Öpik-Wetherill method was used in both these studies, but they differed in their models of the Mars' crossing population. Ivanov (2001) used the observed bright MCOs $(H<15)$ while Le Feuvre \& Wieczorek (2008) used the debiased model of NEOs (Bottke et al. 2002) combined with the observed population of bright MCOs $(H<15)$. These source populations differ slightly from ours; we used the significantly updated observed bright MCOs with $H<16$ as of June 2014. Possibly the most important difference between our work and these previous studies is that they assumed uniform random distributions of the angular elements of MCOs.

\subsubsection{Mean impact velocity}

The current mean encounter velocities reported by Ivanov (2001) and Le Feuvre \& Wieczorek (2008) are $8.6 \mathrm{~km} / \mathrm{s}$ and $9.1 \mathrm{~km} / \mathrm{s}$, respectively. These are slightly higher than our estimates of $8.5 \mathrm{~km} / \mathrm{s}$ for the case of uniform angular distribution, but lower than our result from the non-uniform angular distribution, $10.1 \mathrm{~km} / \mathrm{s}$. (Note that our estimates of mean encounter velocities are obtained for the bright MCOs, $H<16$, see section 4.2.) The small difference in mean values of encounter velocities is understandable considering that the encounter velocity distribution has two strong peaks (Fig. 10). We remark that the clearly double-peaked velocity distribution of impacts on Mars contradicts the model of a singlepeak velocity distribution which has been widely used in Martian cratering studies (Flynn \& McKay 1990; Williams et al. 2014, and references therein).

\subsubsection{Mean impact flux}

The studies by Ivanov (2001) and Le Feuvre \& Wieczorek (2008) do not quote a direct result for the current impact flux on Mars. Instead, they report the impact flux on the Moon and the ratio of the Mars/Moon impact flux. Here we provide comparisons by making some minor calculations from their reported results.

Ivanov (2001) provides the impact flux of the observed $H<18$ NEOs on the lunar surface, $0.77 \times 10^{-15} \mathrm{~km}^{-2} \mathrm{yr}^{-1}$, and writes that this is $40 \%$ of the total impact flux

(accounting for observational incompleteness of $H<18$ NEOs), which gives $F_{D}(18)=$ 
$6.1 \times 10^{-23} \mathrm{~km}^{-2} \mathrm{~s}^{-1}$. This is consistent with our estimate (in Section 4.4) of the lunar impact flux. Ivanov also tabulates the Mars/Moon impact flux ratio for three different values of Mars' eccentricity (Table 1 in his paper). For the current value of Mars eccentricity of 0.093, his ratio is 4.8. From this we obtain Ivanov's estimate for the current impact flux on Mars as $F(18)=2.9 \times 10^{-22} \mathrm{~km}^{-2} \mathrm{~s}^{-1}$.

Le Feuvre \& Wieczorek (2008) report the lunar impact flux, $1.83 \times 10^{-15} \mathrm{~km}^{-2} \mathrm{yr}^{-1}$, for NEOs larger than $1 \mathrm{~km}$. To convert to the flux of $H<18$ objects, we use the ratio of the number of NEOs brighter than $H=18$ and the number of NEOs larger than $1 \mathrm{~km}$, 960/855, to calculate that Le Feuvre \& Wieczorek (2008)'s result for the lunar impact flux is equivalent to $F_{D}(18)=6.5 \times 10^{-23} \mathrm{~km}^{-2} \mathrm{~s}^{-1}$. These authors also reported the current impact flux ratio of Mars/Moon as 3.23. Thus, we obtain that their estimate of the current impact flux on Mars is equivalent to $F(18)=2.1 \times 10^{-22} \mathrm{~km}^{-2} \mathrm{~s}^{-1}$.

For the mean impact flux on Mars, our result, $F(18)=(1.7-3.0) \times 10^{-22} \mathrm{~km}^{-2} \mathrm{~s}^{-1}$, appears to be similar to the previous estimates (Ivanov 2001; Le Feuvre \& Wieczorek 2008). The near-similarity of these results may appear surprising, because we expect our result to be smaller due to the fact that the non-uniform angular distribution depresses the impact flux, as we showed in Section 4.1. The explanation lies in the different source populations; both previous studies likely used a smaller number of MCOs which is closer to the lower limit of our number estimate, as we describe below.

Ivanov (2001) estimated the population of MCOs $(H<18)$ by multiplying the population ratio of Earth-crossing objects $\left(\operatorname{ECOs}^{1}\right),[N(H<18) / N(H<15)]$, with the number of bright MCOs $N(H<15)$. From his estimate of $40 \%$ observational completeness level of $H<18$ ECOs and his plot of the cumulative number distribution of observed ECOs, we estimate his ratio $[N(H<18) / N(H<15)] \approx 20$. The number of bright ECOs and bright MCOs $(H<15)$ in his work is 155 and 400, respectively, therefore we estimate the total number of MCOs $(H<18)$ in his work is approximately eight thousand. In our model, the population ratio of MCOs $[N(H<18) / N(H<15)]$ is $18-30$, based on the two different $H$-distribution functions of Eq. 1 and Eq. 2, and the number of MCOs $(H<15)$ is 539. This yields our estimated population of MCOs of ten-to-sixteen thousand; this is higher than the estimate of Ivanov (2001). Le Feuvre \& Wieczorek (2008) did not directly calculate the impact flux $F(18)$ on Mars; they just reported the Mars/Moon impact flux ratio calculated from bright asteroids $(H<15)$. Thus, Le Feuvre \& Wieczorek (2008)'s F(18) estimate cited above is based on a population model similar to Eq. 2.

In summary, in Ivanov (2001) and Le Feuvre \& Wieczorek (2008)'s studies there was

\footnotetext{
${ }^{1}$ Ivanov (2001) used NEOs as the terminology instead of ECOs.
} 
a fortuitous cancellation of the effect of a smaller number of the population (which is close to our lower estimates) and the effect of the assumed uniform distribution of the angular elements of MCOs of $H<18$. In our study, we have shown that the non-uniform angular distributions depress the impact flux, but our larger population estimate of MCOs (based on up-to-date data of the minor planets) makes the previous estimates appear to be similar to our $F(18)$ range.

\subsubsection{Mars/Moon impact flux ratios}

The Mars/Moon impact flux ratio for the current epoch was determined by Ivanov (2001) as 4.8. Later, Ivanov (2006) updated their estimate to 4.93 (on the basis of updated observational data of MCOs of $H<17$ and for the current value of Mars' eccentricity), but they noted that this value was uncorrected for observational incompleteness of MCOs; such correction would increase the Mars/Moon ratio. Somewhat later still, Le Feuvre \& Wieczorek (2008) calculated the Mars/Moon impact flux ratio as 3.23 and Le Feuvre \& Wieczorek (2011) calculated a value of 3.20, but neither paper commented on the discrepancy with Ivanov's results. This discrepancy is puzzling because both authors reported consistent results for the long term average value of the Mars/Moon impact flux ratio (taking account of Mars' eccentricity evolution): 2.04 in Ivanov (2001), 3-3.5 in Ivanov (2006), 2.83 in Le Feuvre \& Wieczorek (2008), 2.76 in Le Feuvre \& Wieczorek (2011).

Our study finds that the Mars/Moon impact flux ratio depends on the projectile size of interest, unless the MCOs' $H$ distribution is modelled as a scaled version of that of the ECOs (as in Eq. 2). For kilometer-size MCOs (Section 4.4), we found that the impact flux ratio of Mars/Moon is 2.9-5.0. The higher estimate is based on the data of observed bright MCOs, Eq. 1, while the lower estimate obtains from the MCO population model which is a scaled version of that of ECOs, Eq. 2. Note that these two estimates required a relatively small extrapolation, from just $H=16$ to $H=18$, from the same population number, $N(<16)$. Thus, we think that the uncertainty of the Mars/Moon impact flux ratio in the range, 2.9-5.0, expresses the underlying uncertainty in our current knowledge of the population of kilometer-size MCOs.

The origin of the discrepancy amongst the previous estimates remains unknown to us, but may be simply attributed to the uncertainties in the population estimates of kilometersize MCOs. 


\subsubsection{The flux of small impactors and its seasonal variation}

According to our estimates, Mars suffers 14-160 impacts by $D>1 \mathrm{~m}$ objects per Martian year (see section 4.5). We can compare this result with recent analyses of observations of fresh impact craters identified on the surface of Mars with the imaging data from spacecraft presently orbiting Mars.

If we neglect atmospheric effects and adopt the $\pi$-scaling law for projectile-to-crater size scaling (Holsapple 1993), then meter-size projectiles would produce craters of rim-torim diameter $\sim 9-14 \mathrm{~m}$. For this crater size estimate, we assumed the same parameters as in Williams et al. (2014): projectile density $3700 \mathrm{~kg} \mathrm{~m}^{-3}$, target density $2000 \mathrm{~kg} \mathrm{~m}^{-3}$, yield strength $65 \mathrm{kPa}$, and other properties of dry soils. Because the impact velocity distribution on Mars is double-peaked (see Section 4.2), we assumed two values of typical impact velocities, 6.5 and $11.5 \mathrm{~km} / \mathrm{s}$; and we adopted impact angles in the range $30^{\circ}-60^{\circ}$. Thus, our results imply that craters of diameter $D \gtrsim(9-14) \mathrm{m}$ are produced at a rate of $14-160$ craters per Martian year. Daubar et al. (2013) report the current observed Martian cratering rate as $3 \times 10^{-7} \mathrm{~km}^{-2} \mathrm{yr}^{-1}$ for crater diameters $D_{c}>11 \mathrm{~m}$ (Fig. 4 in their paper); this is equivalent to about 80 impacts per Martian year. This agrees with our estimates fairly well, considering the large uncertainties in extrapolating from bright MCOs to faint MCOs.

Regarding the seasonal variations, in his pioneering study, Ivanov (2001) estimated that the impact flux on Mars would have a factor of 7 enhancement when the planet was near aphelion compared to when it was near perihelion. (In order to obtain this factor, he calculated the impact rate by MCOs whose perihelion distances are less than the aphelion distance of Mars, $1.67 \mathrm{AU}$, and ratioed this result to the impact rate by MCOs whose perihelion distances are less than the perihelion distance of Mars, 1.38 AU; B. Ivanov 2015, personal communication.) No other studies were carried out on this topic for more than a decade. In the present study, we have shown that the peak-to-peak seasonal variation of the impact flux of the bright MCOs should be about 11 if their angular elements are uniformly distributed, as Ivanov (2001) assumed, but accounting for the demonstrated intrinsic nonuniformity of the angular elements reduces this factor to $\sim 3$. Integrated over half a Martian year, we find that the aphelion-to-perihelion enhancement of the impact flux of bright MCOs should be 2.1. If the small impactors track the orbital distribution of the bright MCOs and their $H$-distributions are also correlated with inclination, as in Eq. 1, then their impact flux would be dominated by the low inclination population, as discussed in Section 4.5. Our calculations indicate that the peak-to-peak seasonal variation of the flux of small impactors $(D>1 \mathrm{~m}, H<33)$ should be about 3 ; but it could be as large as 11-15 if the angular elements of the small impactors were uniformly distributed.

Daubar et al. (2012) have analyzed the dataset of fresh small Martian craters, and have 
made a preliminary estimate that the aphelion-to-perihelion impact flux ratio, integrated over half a Martian year, is about 1.2. This estimate has large and unspecified uncertainty, but it is closer to our prediction for the case of non-uniform angular elements of meter-size MCOs (Table 2). Daubar et al. (2012) also rule out an aphelion enhancement factor $>3.1$ with $97 \%$ confidence. Comparing with our prediction of a factor of 5.4-6.6 enhancement in the case of the uniform angular distribution, we can tentatively rule out a uniform angular distribution of MCOs.

The time-resolution of the current dataset of fresh Martian craters is insufficient to test our detailed predictions for the peak-to-peak seasonal variation (see Table 2 and Fig. 8). Continuing efforts towards detecting fresh impacts on the Martian surface will provide not

only a better estimate of the mean impact flux but also reveal the orbital distribution of meter-size objects, particularly their non-uniformity in angular elements.

\subsection{Variation of impact flux on secular timescales}

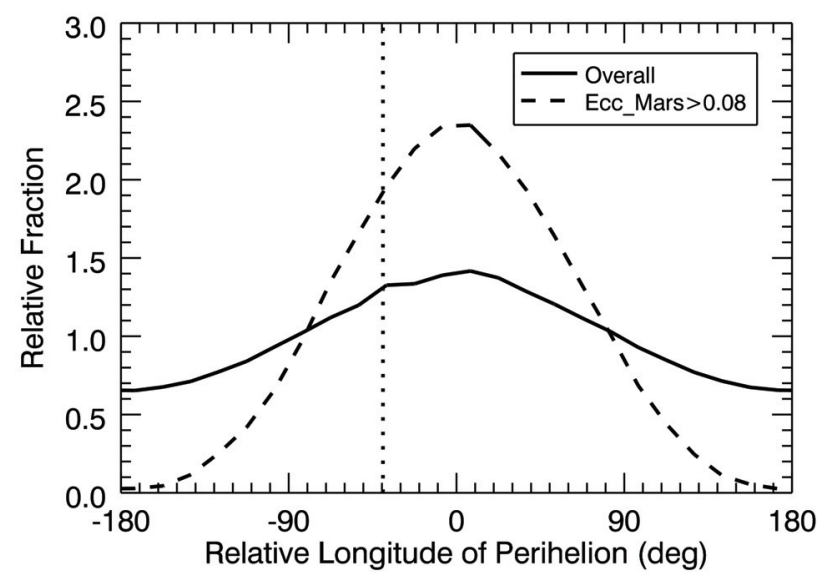

Fig. 12. - The time-weighted distribution of the longitude of Mars' perihelion relative to Jupiter's, $\varpi_{0^{\nearrow}}-\varpi_{4}$, in two different regimes of Mars' eccentricity. The calculation was done with an orbital integration of all eight planets for $10^{8}$ years. The vertical dotted line indicates the current value of $\varpi_{O^{7}}-\varpi_{4}$.

The impact flux estimates described above are specific to the present orbital configuration of Mars. Over secular timescales, the mean impact flux evolves as Mars' orbit evolves, most importantly Mars' eccentricity vector relative to the mean eccentricity vector of MCOs 


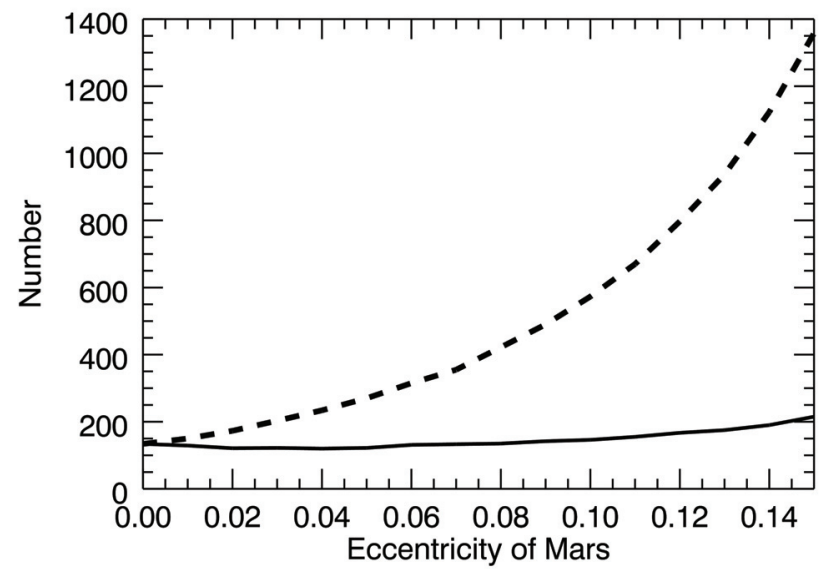

Fig. 13. - Number of Mars crossers $(H<15)$ for different eccentricities of Mars. We use the current orbital distribution of asteroids. The dashed line indicates the result when we use the conventional definition of MCO: the heliocentric distance of an MCO should overlap Mars' heliocentric distance, for given Mars' eccentricity. The solid line indicates the result when we take account of the longitude of perihelion to count only the objects that actually intersect Mars' orbit. (We neglect the inclinations, and we choose the direction of $\varpi_{4}$ for the representative direction of Mars' perihelion longitude.)

(or relative to the orientation of Jupiter's perihelion direction). Previous investigations of the impact flux on Mars averaged over secular timescales have considered only the secular variation of the magnitude of Mars' eccentricity but not its $\varpi$ (Ivanov 2001; Ivanov et al. 2002; Le Feuvre \& Wieczorek 2008). However, as we demonstrated in Section 4, the perihelion orientation is also important, just as much as the magnitude of its eccentricity. For example, when Mars' $\varpi$ is anti-aligned with Jupiter while the MCOs' eccentricity vectors tend to align with the $\varpi$ of Jupiter, the impact rate on Mars would be greater than the value calculated from the uniform angular distribution of MCOs. Therefore, it is important to account for the coupled eccentricity and apsidal variations to calculate the time-averaged impact flux on Mars.

Some of the Mars crossing population of asteroids is also subject to the dynamical effects of mean motion resonances with Mars (Morbidelli et al. 2002; Nesvorný et al. 2002). Although these resonances are not long term stable, they may provide some degree of protection from collision with Mars on timescales similar to secular timescales. This effect has not been included in previous studies nor in the present work, but should be included in future studies. 
A full investigation of the effect of the non-uniform angular variables on the variation of the impact flux on Mars over secular timescales is beyond the scope of the present paper. However, we make a few preliminary notes. We carried out a numerical integration of the orbital evolution of the eight major planets (Mercury-Neptune) for $10^{8}$ years, with initial conditions at the present epoch. We observe that the evolution of Mars' eccentricity and its longitude of perihelion relative to Jupiter's, $\varpi_{\sigma^{\top}}-\varpi_{4}$, are highly correlated; this is shown in Figure 12. The overall time-weighted distribution of $\varpi_{\sigma^{\pi}}$ has a clear concentration near $\varpi_{4}$ (as indicated by the peak near zero of the solid line). Moreover, during the epochs when the eccentricity of Mars is high, exceeding 0.08, we observe that $\varpi_{\sigma^{\pi}}$ is even more highly concentrated near Jupiter's and is rarely found anti-aligned with that planet. The duration of epochs when Mars has high eccentricity (in excess of 0.08 ) account for $28 \%$ of the whole $10^{8} \mathrm{yr}$ simulation period. The present-day Martian eccentricity, 0.093, lies within this high eccentricity range. (We note that the probability of anti-alignment of Mars' perihelion relative to Jupiter's is vanishingly small when Mars' eccentricity exceeds 0.093, i.e., its current value.)

A large fraction of the current Mars-crossing population of minor planets is located near the inner edge of the main asteroid belt. As Mars eccentricity changes over secular timescales, the population of MCOs changes by definition, as the perihelion and aphelion cut-off distances must overlap Mars' heliocentric distance range. The number of MCOs is a steeply increasing function of Mars' eccentricity, because a larger aphelion distance of Mars includes larger numbers of minor planets as Mars-crossers. Thus, considering that Mars' current eccentricity is closer to its secular maximum, it is tempting to conclude that the long time average impact flux on Mars is significantly lower than its current value. For example, Ivanov (2001) estimated that the time-averaged impact flux on Mars is less than 50\% of its present-day impact flux. The true answer is not straightforward to determine if we take account of the non-uniform distribution of the angular elements of the minor planets which can significantly affect the number of potential impactors which depending on the relative orbital geometries, as illustrated in Fig. 6. For example, in the planar approximation, the number of MCOs would be the same as the number of intersecting orbits when Mars' orbit is circular; however, for non-zero eccentricity of Mars, the preference for alignment of $\varpi$ of MCOs with $\varpi_{4}$ reduces the number of intersecting orbits, hence potential impactors. To examine this, we made a rough calculation of how the number of MCOs $(H<15)$ varies with Mars' eccentricity. (We set the perihelion longitude of Mars equal to $\varpi_{4}$, since this is the most probable case, especially for highly eccentric Mars, cf. Fig. 12.) The results are shown in Fig. 13. The dashed line in this figure shows the number of MCOs $(H<$ 15) when we adopt the same definition of MCOs as in Section 2.1, i.e., the heliocentric distance range of an $\mathrm{MCO}$ overlaps the heliocentric distance range of Mars, for specified 
Mars' eccentricities; as expected, this number increases tremendously with increasing Mars' eccentricity. However, when we take account of the angular elements and count the number of minor planets whose orbits actually intersect Mars orbit (as illustrated in Fig. 6), we get the curve shown as the solid line in Fig. 13; this curve is nearly horizontal, indicating only a small change in the number of potential impactors as a function of Mars' eccentricity. Although a direction of $\varpi_{\sigma^{\pi}}$ differing from $\varpi_{4}$ could increase the number of intersecting orbits above that shown in the solid line in Fig. 13, it is clear that the time-weighted values would not be dramatically different than that of the current number of MCOs, because $\varpi_{\sigma^{\pi}}$ is restricted to the neighborhood of $\varpi_{4}$ for highly eccentric Mars.

Thus, we cautiously predict that the long time averaged impact flux on Mars' may not be as strongly different from its current value as Ivanov (2001) claimed, because the current near-alignment of Mars' $\varpi$ with Jupiter's reduces the impact flux compared to the random angle distribution assumed in Ivanov's calculations. Furthermore, the simulation results obtained by Le Feuvre \& Wieczorek (2008), who also assumed random distributions of angular elements, find that the current impact flux is just $14 \%$ higher than the long time average value. Therefore, if the effect of the non-uniform angular distribution of MCOs were included, we cannot rule out the possibility that the current impact flux is even lower than the time-averaged flux over secular timescales.

\section{Summary}

In this work, we have provided an updated and detailed calculation of the current impact flux on Mars, including its seasonal variation. We also provide an updated estimate for the Mars/Moon, Earth/Moon and Mars/Earth impact flux ratios. We use a modification of the method of Öpik (1951) and Wetherill (1967) for the collision probability of two independent Keplerian orbits. Our impact flux calculation is based on the observationally nearly-complete population of bright Mars-crossing objects (respectively, Earth-crossing objects, for the lunar and terrestrial flux). We pay careful attention to the non-uniform distribution of angular elements of this population and the effects of gravitational focusing. We show that this non-uniform distribution, which is owed to the secular perturbations of the planets, strongly affects the mean impact flux on Mars as well as its seasonal variation. It also affects the velocity distribution of impacts on Mars. We also provide predictions for the meter-size impact flux on Mars, including its seasonal variation; we note that the magnitude of its seasonal variation is very sensitive to the orbital distribution, particularly the non-uniformity of the angular elements. Observations of fresh Martian craters can test these predictions, and help to reveal the population and orbital distribution of the small size Mars-crossing 
objects. A summary of our results is as follows.

1. The population of Mars crossing objects has a strongly non-uniform distribution of the longitude of perihelion and the argument of perihelion, owing to secular planetary perturbations. The longitude of perihelion has unidirectional distribution, approximately aligned with Jupiter's eccentricity vector. The argument of perihelion has an axial distribution, with concentrations at $90^{\circ}$ and $270^{\circ}$.

2. The current mean impact flux on Mars by asteroids brighter than $H=16$ is $2.9 \times$ $10^{-23} \mathrm{~km}^{-2} \mathrm{~s}^{-1}$. If the non-uniform distribution of the angular elements of MCOs were to be neglected, this flux would be about twice as large.

3. The impact flux of bright objects $(H<16)$ on Mars has a strong seasonal variation: it is at a maximum at Mars aphelion and minimum at its perihelion. In the current era, the impact flux $\pm 30^{\circ}$ near aphelion is about three times the impact flux near perihelion. If the non-uniform distribution of the angular elements of MCOs were to be neglected, this enhancement factor would be about eleven.

4. The impact velocity distribution has two distinct peaks, at $6.5 \mathrm{~km} \mathrm{~s}^{-1}$ and $11.5 \mathrm{~km} \mathrm{~s}^{-1}$, and a mean value of $11.5 \mathrm{~km} \mathrm{~s}^{-1}$. It also has a strong seasonal variation, with a minimum at Mars' aphelion and a maximum at its perihelion.

5. The Mars/Moon impact flux ratio is 2.9-5.0, and the Mars/Earth ratio is 1.7-2.8 for kilometer size objects. Our updated estimate for the current impact flux on the Moon and Earth by minor planets brighter than $H=18$ is $6 \times 10^{-23} \mathrm{~km}^{-2} \mathrm{~s}^{-1}$ and $1.0 \times 10^{-22} \mathrm{~km}^{-2} \mathrm{~s}^{-1}$, respectively. The Earth/Moon impact flux ratio is 1.7 .

6. The flux of small impactors, $H<33$ (approximately meter-size) objects, on Mars is $(1.6-19) \times 10^{-15} \mathrm{~km}^{-2} \mathrm{~s}^{-1}$, or roughly $14-160$ impacts per martian year. If the small impactors track the orbital distribution of the bright objects, then we predict that their impact flux near Mars' aphelion $\left( \pm 30^{\circ}\right)$ is three times that near perihelion $\left( \pm 30^{\circ}\right)$. However if their angular elements have a uniform distribution, then this enhancement factor would be 11-15. Integrated over half a Martian year, we predict the aphelion-to-perihelion impact flux enhancement is a factor of $\sim 2$ for the case of non-uniform angular elements and about 5.4-6.6 for the case of uniform angular distribution. Comparing with the current dataset of fresh impact craters on Mars, we can tentatively rule out the latter.

The algorithms and computer codes used in this work are available upon request from the authors. 
We thank David P. O'Brien, William F. Bottke, Boris A. Ivanov, and Ingrid J. Daubar for helpful suggestions and discussions. This research was supported by NSF (grant AST1312498) and NASA (grant NNX14AG93G), and made use of the NASA Astrophysics Data System Bibliographic Services and the Minor Planet Center database.

\section{REFERENCES}

Bottke, W. F., and R. Greenberg 1993. Asteroidal collision probabilities. Geophys. Res. Lett. 20, 879-881.

Bottke, W. F., A. Morbidelli, R. Jedicke, J.-M. Petit, H. F. Levison, P. Michel, and T. S. Metcalfe 2002. Debiased Orbital and Absolute Magnitude Distribution of the NearEarth Objects. Icarus 156, 399-433.

Brown, P., R. E. Spalding, D. O. ReVelle, E. Tagliaferri, and S. P. Worden 2002. The flux of small near-Earth objects colliding with the Earth. Nature 420, 294-296.

Brown, P. G., J. D. Assink, L. Astiz, R. Blaauw, M. B. Boslough, J. Borovička, N. Brachet, D. Brown, M. Campbell-Brown, L. Ceranna, W. Cooke, C. de Groot-Hedlin, D. P. Drob, W. Edwards, L. G. Evers, M. Garces, J. Gill, M. Hedlin, A. Kingery, G. Laske, A. Le Pichon, P. Mialle, D. E. Moser, A. Saffer, E. Silber, P. Smets, R. E. Spalding, P. Spurný, E. Tagliaferri, D. Uren, R. J. Weryk, R. Whitaker, and Z. Krzeminski 2013. A 500-kiloton airburst over Chelyabinsk and an enhanced hazard from small impactors. Nature 503, 238-241.

Cañada-Assandri, M., R. Gil-Hutton, and A. O. Ribeiro 2015. The Hungaria population: A comparison between sub-groups. Planet. Space Sci. 105, 60-64.

Chapman, C. R. 2004. Mars Cratering Issues: Secondary Cratering and End-Noachian Degradation. In S. Clifford, J. Farmer, R. Haberle, H. Newsom, and T. Parker (Eds.), Second Conference on Early Mars: Geologic, Hydrologic, and Climatic Evolution and the Implications for Life, pp. 8028.

Daubar, I. J., M. P. Golombek, A. S. McEwen, S. Byrne, M. Kreslavsky, N. C. Schmerr, M. E. Banks, P. Lognonné, T. Kawamura, and F. Karakostas 2015. Measurement of the Current Martian Cratering Size Frequency Distribution, Predictions for and Expected Improvements from InSight. In Lunar and Planetary Science Conference, Volume 46 of Lunar and Planetary Inst. Technical Report, pp. 2468. 
Daubar, I. J., A. S. McEwen, S. Byrne, and M. R. Kennedy 2012. Seasonal Variation in Current Martian Impact Rate. In Lunar and Planetary Science Conference, Volume 43 of Lunar and Planetary Inst. Technical Report, pp. 2740.

Daubar, I. J., A. S. McEwen, S. Byrne, M. R. Kennedy, and B. Ivanov 2013. The current martian cratering rate. Icarus 225, 506-516.

Dell'Oro, A., F. Marzari, P. Paolicchi, and V. Vanzani 2001. Updated collisional probabilities of minor body populations. A\&A 366, 1053-1060.

Dell'Oro, A., and P. Paolicchi 1998. Statistical Properties of Encounters among Asteroids: A New, General Purpose, Formalism. Icarus 136, 328-339.

Farinella, P., D. Vokrouhlický, and W. K. Hartmann 1998. Meteorite Delivery via Yarkovsky Orbital Drift. Icarus 132, 378-387.

Fisher, N. 1993. Statistical Analysis of Circular Data. Cambridge University Press.

Flynn, G. J., and D. S. McKay 1990. An assessment of the meteoritic contribution to the Martian soil. J. Geophys. Res. 95, 14497-14509.

Greenberg, R. 1982. Orbital interactions - A new geometrical formalism. AJ 87, 184-195.

Gronchi, G. F. 2005. An Algebraic Method to Compute the Critical Points of the Distance Function Between Two Keplerian Orbits. Celestial Mechanics and Dynamical Astronomy 93, 295-329.

Halliday, I., A. A. Griffin, and A. T. Blackwell 1996. Detailed data for 259 fireballs from the Canadian camera network and inferences concerning the influx of large meteoroids. Meteoritics and Planetary Science 31, 185-217.

Harris, A. W. 2002. A New Estimate of the Population of Small NEAs. In AAS/Division for Planetary Sciences Meeting Abstracts \#34, Volume 34 of Bulletin of the American Astronomical Society, pp. 835.

Hartmann, W. K. 2005. Martian cratering 8: Isochron refinement and the chronology of Mars. Icarus 174, 294-320.

Hartmann, W. K., and G. Neukum 2001. Cratering Chronology and the Evolution of Mars. Space Sci. Rev. 96, 165-194.

Hartmann, W. K., C. Quantin, S. C. Werner, and O. Popova 2010. Do young martian ray craters have ages consistent with the crater count system? Icarus 208, 621-635. 
Holsapple, K. A. 1993. The scaling of impact processes in planetary sciences. Annual Review of Earth and Planetary Sciences 21, 333-373.

Ito, T., and K. Tanikawa 2002. Long-term integrations and stability of planetary orbits in our Solar system. MNRAS 336, 483-500.

Ivanov, B. A. 2001. Mars/Moon Cratering Rate Ratio Estimates. Space Sci. Rev. 96, 87-104.

Ivanov, B. A. 2006. Cratering Rate Comparisons Between Terrestrial Planets. LPI Contributions 1320, 26-27.

Ivanov, B. A., G. Neukum, W. F. Bottke, Jr., and W. K. Hartmann 2002. The Comparison of Size-Frequency Distributions of Impact Craters and Asteroids and the Planetary Cratering Rate. Asteroids III, 89-101.

JeongAhn, Y., and R. Malhotra 2014. On the non-uniform distribution of the angular elements of near-Earth objects. Icarus 229, 236-246.

Kozai, Y. 1962. Secular perturbations of asteroids with high inclination and eccentricity. AJ 67, 591.

Laskar, J., A. C. M. Correia, M. Gastineau, F. Joutel, B. Levrard, and P. Robutel 2004. Long term evolution and chaotic diffusion of the insolation quantities of Mars. Icarus 170, 343-364.

Le Feuvre, M., and M. A. Wieczorek 2008. Nonuniform cratering of the terrestrial planets. Icarus 197, 291-306.

Le Feuvre, M., and M. A. Wieczorek 2011. Nonuniform cratering of the Moon and a revised crater chronology of the inner Solar System. Icarus 214, 1-20.

Mainzer, A., T. Grav, J. Bauer, J. Masiero, R. S. McMillan, R. M. Cutri, R. Walker, E. Wright, P. Eisenhardt, D. J. Tholen, T. Spahr, R. Jedicke, L. Denneau, E. DeBaun, D. Elsbury, T. Gautier, S. Gomillion, E. Hand, W. Mo, J. Watkins, A. Wilkins, G. L. Bryngelson, A. Del Pino Molina, S. Desai, M. Gómez Camus, S. L. Hidalgo, I. Konstantopoulos, J. A. Larsen, C. Maleszewski, M. A. Malkan, J.-C. Mauduit, B. L. Mullan, E. W. Olszewski, J. Pforr, A. Saro, J. V. Scotti, and L. H. Wasserman 2011. NEOWISE Observations of Near-Earth Objects: Preliminary Results. Apj 743, 156.

Malin, M. C., K. S. Edgett, L. V. Posiolova, S. M. McColley, and E. Z. N. Dobrea 2006. Present-Day Impact Cratering Rate and Contemporary Gully Activity on Mars. Science 314, 1573-. 
Michel, P., F. Migliorini, A. Morbidelli, and V. Zappalà 2000. The Population of MarsCrossers: Classification and Dynamical Evolution. Icarus 145, 332-347.

Morbidelli, A., W. F. Bottke, Jr., C. Froeschlé, and P. Michel 2002. Origin and Evolution of Near-Earth Objects. Asteroids III, 409-422.

Morbidelli, A., R. Jedicke, W. F. Bottke, P. Michel, and E. F. Tedesco 2002. From Magnitudes to Diameters: The Albedo Distribution of Near Earth Objects and the Earth Collision Hazard. Icarus 158, 329-342.

Murray, C. D., and S. F. Dermott 1999. Solar system dynamics.

Nesvorný, D., S. Ferraz-Mello, M. Holman, and A. Morbidelli 2002. Regular and Chaotic Dynamics in the Mean-Motion Resonances: Implications for the Structure and Evolution of the Asteroid Belt. Asteroids III, 379-394.

O'Brien, D. P., and R. Greenberg 2005. The collisional and dynamical evolution of the main-belt and NEA size distributions. Icarus 178, 179-212.

Öpik, E. J. 1951. Collision probabilities with the planets and the distribution of interplanetary matter. Proc. R. Irish Acad. Sect. A, vol. 54, p. 165-199 (1951). 54, 165-199.

Pokorný, P., and D. Vokrouhlický 2013. Öpik-type collision probability for high-inclination orbits: Targets on eccentric orbits. Icarus 226, 682-693.

Rabinowitz, D., E. Helin, K. Lawrence, and S. Pravdo 2000. A reduced estimate of the number of kilometre-sized near-Earth asteroids. Nature 403, 165-166.

Revelle, D. O. 2001. Global infrasonic monitoring of large bolides. In B. Warmbein (Ed.), Meteoroids 2001 Conference, Volume 495 of ESA Special Publication, pp. 483-489.

Stuart, J. S., and R. P. Binzel 2004. Bias-corrected population, size distribution, and impact hazard for the near-Earth objects. Icarus 170, 295-311.

Terai, T., and Y. Itoh 2011. Size Distribution of Main-Belt Asteroids with High Inclination. PASJ 63, 335-346.

Warner, B. D., A. W. Harris, D. Vokrouhlický, D. Nesvorný, and W. F. Bottke 2009. Analysis of the Hungaria asteroid population. Icarus 204, 172-182.

Wetherill, G. W. 1967. Collisions in the Asteroid Belt. J. Geophys. Res. 72, 2429.

Wetherill, G. W. 1974. Problems Associated with Estimating the Relative Impact Rates on Mars and the Moon. Moon 9, 227-231. 
Williams, J.-P., A. V. Pathare, and O. Aharonson 2014. The production of small primary craters on Mars and the Moon. Icarus 235, 23-36. 